\title{
Relativistic Mean-Field Models with Effective Hadron Masses and Coupling Constants, and $\rho^{-}$Condensation
}

\author{
E.E.Kolomeitsev ${ }^{\mathrm{a}}$ and D.N. Voskresensky ${ }^{\mathrm{b}, \mathrm{c}}$ \\ ${ }^{a}$ Niels Bohr Institute, Blegdamsvej 17, Copenhagen, Denmark \\ ${ }^{\mathrm{b}}$ Moscow Engineering Physical Institute, \\ Kashirskoe Avenue 31, RU-11549 Moscow, Russia \\ ${ }^{\mathrm{c}}$ GSI, Plankstraße 1, D-64291 Darmstadt, Germany
}

\begin{abstract}
We study relativistic mean-field models with hadron masses and coupling constants depending self-consistently on a scalar meson field. We demonstrate that by the field redefinition some models can be equivalently transformed into each other. Thereby the large variety of scaling functions for masses and couplings can be reduced to a restricted set of functions with a constrained dependence on a scalar field. We show how by choosing properly the latter scaling functions one may stiffen or soften the equation of state at high densities and simultaneously increase the threshold density for the direct Urca process without any change of the description of nuclear matter close to the saturation density. The stiffening of the equation of state might be motivated by recent neutron star mass measurements, whereas the increase of the threshold density for the direct Urca process is required by the analysis of neutron star cooling data. We demonstrate that if a rho meson is included in a mean-field model as a non-Abelian gauge boson, then there is a possibility for a charged rho-meson condensation in dense nuclear matter. We show that such a novel phase can be realized in neutron star interiors already for sufficiently low densities, typically $\sim 3 \div 4 n_{0}$, where $n_{0}$ is the nuclear saturation density. In the framework of the relativistic mean field model the new phase arises in a secondorder phase transition. The appearance of a $\rho^{-}$condensate significantly alters the proton fraction in a neutron star but changes moderately the equation of state. The neutrino emissivity of the processes involving a $\rho^{-}$meson condensate is estimated.
\end{abstract}




\section{Introduction}

The baryon matter at densities relevant for neutron star (NS) interiors is convenient to describe in terms of relativistic mean-field (RMF) models, cf. [1] and references therein. In these models the nucleon mass decreases with an increase of the nucleon density due to coupling to a scalar field, whereas the effective masses of scalar $(\sigma)$, vector $(\omega)$ and isovector $(\rho)$ mesons, and their coupling constants, are usually assumed to be density and field independent quantities. The RMF models provide a simple and economical tool for construction of the equation of state (EoS) for dense baryonic matter. By tuning parameters of a RMF model at a saturation density one may get an EoS similar to the one that follows from more involved microscopic calculations, e.g.,

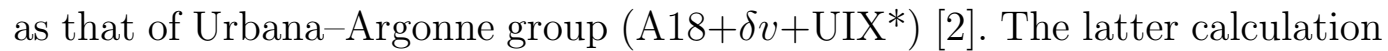
provides probably the most realistic EoS for nuclear densities up to $4 n_{0}$, but it uses a non-relativistic potential and violates causality at higher densities. As an extrapolation to higher densities RMF models are practical. However all RMF models have the very same an unpleasant feature. They produce a large fraction of protons in the NS matter, cf. [3,4]. This permits a very efficient cooling process, direct Urca (DU) process $n \rightarrow p e \bar{\nu}$, for $n>n_{\text {crit }}^{\mathrm{DU}}$, with a low threshold density $n_{\text {crit }}^{\mathrm{DU}} \lesssim 3 n_{0}$. Ref. [5] emphasized this fact as an obvious shortcoming of RMF models. Oppositely, ref. [6] and some subsequent works [7] tried to use this fact to develop a so-called DU-based scenario of a NS cooling (based on $\left.n_{\text {crit }}^{\mathrm{DU}} \sim(2 \div 3) n_{0}\right)$.

The NS cooling "data" (surface temperatures of NSs as a function of the NS age) can be subdivided in three groups, corresponding to "slow cooling", "intermediate cooling" and "rapid cooling" of neutron stars. If the density in the NS central region exceeds $n_{\text {crit }}^{\mathrm{DU}}$, which corresponds to the mass $M_{\text {crit }}^{\mathrm{DU}}$, the star cools down very fast, radiating mostly from its central region. Thus, the NSs with $M<M_{\text {crit }}^{\mathrm{DU}}$ cool slowly, whereas the stars with the mass $M$ only slightly above $M_{\text {crit }}^{\text {DU }}$ would be already characterized by a very rapid cooling. Such a scenario was criticized in $[8,9,10]$. It is doubtful that many NSs, belonging to an intermediate cooling group, have the very similar masses. It has been already experimentally established that NSs may have essentially different masses. E.g., masses of two NSs in double NS systems are measured with the very high precision, $M_{\mathrm{B} 1913+16}=1.4408 \pm 0.0003 M_{\odot}$, and $M_{\mathrm{J} 0737-3039 \mathrm{~B}}=1.250 \pm 0.005 M_{\odot}[12]$.

Authors of many RMF models do not care about the DU threshold at all and obtain so low values of $n_{\text {crit }}^{\mathrm{DU}}$ that $M_{\text {crit }}^{\mathrm{DU}}<1 M_{\odot}$. However according to the standard mechanisms only the NSs with $M \gtrsim 1 M_{\odot}$ can be formed in supernova explosions. The mentioned above microscopic Urbana-Argonne model produces $n_{\text {crit }}^{\mathrm{DU}} \simeq 5 n_{0}$, that corresponds to a heavy NS with $M_{\text {crit }}^{\mathrm{DU}} \simeq 2 M_{\odot}$. If one used a standard RMF model fitted in [11] to reproduce the Urbana- 
Argonne EoS for $n<4 n_{0}$, but, as well as other RMF models, producing a low DU threshold $\left(n_{\text {crit }}^{\mathrm{DU}} \simeq(2.6 \div 2.7) n_{0}\right)$, one would conclude that the majority of the NSs seen in soft $X$ rays are low mass objects with the mass $M<1.3 M_{\odot}$. The latter value is however below the averaged value of the NS mass $\left(M \simeq 1.35 \pm 0.04 M_{\odot}\right)$ measured in NS binaries [13].

Another problem is that there might exist heavy NSs. Recent measurements of NS masses in binary compact systems yielded $M_{\mathrm{J} 0751+1807}=2.2 \pm 0.2 M_{\odot}$ [12] and $M_{4 \mathrm{U} 1700-37}=2.44 \pm 0.27 M_{\odot}$ [14]. The latter object might be a black hole due to a lack of pulsation but the former object, J0751+1807, is definitely a NS. The confidence level of $95 \%$ still allows to have a smaller limiting NS mass than $2 M_{\odot}$ (however the limiting NS mass is surely higher than $\left.1.6 M_{\odot}\right)$. Additional information on heavy NSs may be obtained from quasi-periodic oscillation (QPO) sources. These are NSs that are $X$-ray sources at frequencies of the orbiting accreting matter. If QPOs originate from the innermost stable orbit [15] of the accreting matter, their observed values imply that the accreting NS has the mass $\simeq 2.2 M_{\odot}$ in the case of $4 \mathrm{U} 1820-30$. The value $2.2 M_{\odot}$ is the limiting mass of a NS following the Urbana-Argonne EoS. Unfortunately this EoS is acausal at high densities. Ref. [16] suggested an analytic fit of the Urbana-Argonne EoS for $n \lesssim 4 n_{0}$, which respects causality for larger densities. We will denote this parameterization as the HHJ EoS. As a price, the limiting mass decreased down to $\simeq 2 M_{\odot}$. Moreover, all phase transitions, like pion and kaon condensations, and quark matter, which are assumed to be possible in NS interiors, may essentially soften EoS decreasing further the limiting mass of a $\mathrm{NS}^{1}$. Thus modern microscopically motivated EoS and possible phase transitions in NS interiors depend crucially on one precision measurement of a heavy NS with $M>(2 \div 2.2) M_{\odot}$.

Within a standard RMF model it would be hard to describe heavy NSs with $M>2.2 M_{\odot}$ without either an essential increase of the compressibility parameter $K$ or a decrease of the effective nucleon mass at the saturation density. Both values are rather constrained by the atomic nucleus data. The problem originates from the fact that the RMF model parameters are fixed at the saturation density and no free parameters remain to control the pressure and the symmetry energy at higher densities. The problem could be in principle solved by introducing new terms with new fitting parameters.

There are strong indications that meson masses as well as the nucleon mass depend on the nucleon density. A general trend for such a dependence is governed by the property that masses of all hadrons except Goldstone bosons,

1 The Urbana-Argonne calculation [2] already includes neutral pion condensate for $n>1.3 n_{0}$ that only slightly softens EoS in their case. 
like pions and kaons, ${ }^{2}$ should decrease, when the nucleon density $n$ increases towards a critical point of the chiral symmetry restoration [18]. According to the conjecture of Brown and Rho [19] the nucleon mass and the masses of vector $\omega, \rho$ and scalar $\sigma$ mesons may obey an approximately universal scaling law (a so-called "Brown-Rho scaling")

$$
\frac{m_{\rho}^{*}}{m_{\rho}} \simeq \frac{m_{\omega}^{*}}{m_{\omega}} \simeq \frac{m_{\sigma}^{*}}{m_{\sigma}} \simeq \frac{m_{N}^{*}}{m_{N}}=\Phi_{B R}
$$

where asterisks indicate in-medium masses. According to [19] $\Phi_{B R}$ decreases with the increase of the density. In ref. [20] an attempt was made to include density dependent masses in the RMF model in order to incorporate the Brown-Rho scaling, explicitly. A theoretical disadvantage of this model is that the density dependence, $\Phi_{B R} \simeq\left(1+y n / n_{0}\right)^{-1}, y \simeq 0.28$, was inserted by hands into the model Lagrangian rather than it followed from the corresponding equations of motion. Insertion of a density dependence into parameters of a mean-field Lagrangian violates conservation of the energy-momentum and spoils the thermodynamic consistency. Therefore, such models require a special treatment [21]. Ref. [20] also realized that the scaling of meson masses (1) would result in a dramatic stiffening of the EoS, if one does not simultaneously scale the corresponding coupling constants in a similar way. As an argument for such a procedure, authors relied on the description of the flow in heavy ion collisions. Below we will show that this prescription relies on equivalence of some RMF models that allows to use different interpolating mean fields without changing physical quantities on a mean-field level in homogeneous matter.

In ref. [22] the density dependence of effective hadronic masses was incorporated into the mean-field Lagrangian via extra terms describing a hadron interaction with a scalar field. It resulted, however, in an increase of the $\rho$ and $\omega$ meson masses with increase of the nucleon density in disagreement with (1). At the same time the $\sigma$ mass was assumed to be constant. Thus, the inclusion of the hadron mass scaling into RMF models deserves a further consideration. Some other works, cf. [23], played with a field-dependent parameters of generalized mean field models, permitting changes of both masses and couplings. Equivalence between different parameter choices was not realized in these papers.

Appropriate knowledge of the EoS of hot, dense, and isospin asymmetrical nuclear matter is essential for understanding the results of heavy-ion collisions. Flow, particle yields, fragmentation may give some constraints on the EoS and the symmetry energy, cf. $[24,25]$. Often these constraints disagree with those

$\overline{2}$ The kaon effective mass may also decrease with the density, whereas the nature of this decrease is different $[17,1,11]$. 
obtained from NS physics, e.g., yielding very low DU threshold density. The proper choice of the EoS is particularly important for the CBM (compressed baryon matter) experimental program at the future facility at GSI. Precision measurements of heavy ion collisions are planned in the whole energy range from $\sim 1 \mathrm{GeV} / \mathrm{A}$ up to $\sim 40 \mathrm{GeV} / \mathrm{A}$.

To describe correctly the symmetry energy of the nucleon matter one needs to include the $\rho$-meson field into a mean-field Lagrangian. The $\rho$-meson field is traditionally assumed to have only one mean-field component, $\left(\vec{\rho}_{\mu}\right)^{a}=$ $\delta_{\mu 0} \delta^{a 3} \rho_{0}^{(3)}$, where $\mu=0,1,2,3$ is the Lorentz index, and $a=1,2,3$ is the isospin index. This field produces then the expected contribution to the energy density $\propto\left(n_{p}-n_{n}\right)^{2}$, where $n_{p}$ and $n_{n}$ are the proton and neutron densities. In ref. [26] it was realized that, if the $\rho$-meson is introduced as a non-Abelian gauge boson, e.g., in the framework of the hidden local symmetry model [27], then at a density $n>n_{\mathrm{c}}^{\rho}$ the charged $\rho$-meson condensation occurs. In a new phase the symmetry energy grows as $\propto\left|n_{p}-n_{n}\right|$ only. The critical density was found to be $n_{\mathrm{c}}^{\rho} \sim m_{\rho}^{* 3}$, being sensitively dependent on the value of the in-medium mass of the $\rho$-meson $\left(m_{\rho}^{*}\right)$. The phenomenon of the charged $\rho$ meson condensation is similar to the condensation of gauge bosons in QCD [28] (gluon condensate) and to electro-weak [29] ( $W$-boson condensate) sectors of the Standard model.

In this paper we first construct a generalized RMF model with all parameters, being field dependent (secs. 2,3). We derive the energy density functional, and show how various standard cases can be reproduced. We discuss the equivalence relations among some cases. Then we demonstrate shortcomings of the models with a hadron mass scaling without a scaling of coupling constants. We show that the RMF models with the universal scaling for masses and coupling constants can be reduced to the models without any scaling. Such models do not solve, however, the problem of the low $n_{\text {crit }}^{\mathrm{DU}}$. Then we allow for a different scaling of hadron masses and coupling constants in order to generate a model that produces a stiffer EoS at high densities. This model supports a large limiting mass of the NS and rather high critical density for the DU process. On the other hand, it uses appropriate values of compressibility and effective nucleon mass at the saturation nuclear density. Then we demonstrate another example of the RMF model with a non-universal scaling of masses and coupling constants that fits well the HHJ EoS including the correct density dependence of the proton concentration. In sec. 4 we investigate a possibility for a charged $\rho$-meson condensation. We demonstrate that in the framework of our generalized RMF models the charged $\rho$-meson condensation occurs as a second-order phase transition. Emissivity of the NS cooling processes involving the charged rho condensate is estimated in sec. 5. Results are summarized in the concluding section 6. In Appendix we show how one can introduce inhomogeneous electric potential. Throughout the paper we use units $\hbar=c=1$. 


\section{Generalized relativistic mean-field model}

\subsection{Mean field Lagrangian}

We start with a generalized RMF model, where effective hadron masses and coupling constants are assumed to be field-dependent from the very beginning. The Lagrangian density is given by

$$
\begin{aligned}
& \mathcal{L}=\mathcal{L}_{N}+\mathcal{L}_{M}+\mathcal{L}_{l}, \\
& \mathcal{L}_{N}=a_{N} \bar{\Psi}_{N}(i D \cdot \gamma) \Psi_{N}-m_{N} \phi_{N} \bar{\Psi}_{N} \Psi_{N}, \\
& D_{\mu}=\partial_{\mu}+i g_{\omega} \tilde{\chi}_{\omega} \omega_{\mu}+\frac{i}{2} g_{\rho} \tilde{\chi}_{\rho} \vec{\rho}_{\mu} \vec{\tau} \\
& \mathcal{L}_{M}=a_{\sigma} \frac{\partial^{\mu} \sigma \partial_{\mu} \sigma}{2}-\phi_{\sigma}^{2} \frac{m_{\sigma}^{2} \sigma^{2}}{2}-\tilde{U}(\sigma) \\
& -a_{\omega} \frac{\omega_{\mu \nu} \omega^{\mu \nu}}{4}+\phi_{\omega}^{2} \frac{m_{\omega}^{2} \omega_{\mu} \omega^{\mu}}{2}-a_{\rho} \frac{\vec{\rho}_{\mu \nu} \vec{\rho}^{\mu \nu}}{4}+\phi_{\rho}^{2} \frac{m_{\rho}^{2} \vec{\rho}_{\mu} \vec{\rho}^{\mu}}{2}, \\
& \omega_{\mu \nu}=\partial_{\nu} \omega_{\mu}-\partial_{\mu} \omega_{\nu}, \quad \vec{\rho}_{\mu \nu}=\partial_{\nu} \vec{\rho}_{\mu}-\partial_{\mu} \vec{\rho}_{\nu}+g_{\rho}^{\prime} \tilde{\chi}_{\rho}^{\prime}\left[\vec{\rho}_{\mu} \times \vec{\rho}_{\nu}\right] \\
& \mathcal{L}_{l}=\sum_{l} \bar{\Psi}_{l}\left[i(\gamma \cdot \partial)-m_{l}\right] \Psi_{l} .
\end{aligned}
$$

Here $\Psi_{N}=\left(\Psi_{n}, \Psi_{p}\right)^{\mathrm{T}}$ is the isospin doublet of the nucleon bispinors, $\sigma, \omega_{\mu}$, $\vec{\rho}_{\mu}$ are the $\sigma, \omega$ and $\rho$ meson fields, $\vec{\tau}$ is the isospin Pauli matrix. The part $\mathcal{L}_{l}$ describes the contribution of the light leptons (electrons and muons, $l=e, \mu$ ). For the sake of simplicity we will neglect hyperons in the Lagrangian (2), which can contribute, when the hyperon Fermi seas are filled in the NS matter. An extension for hyperons is straightforward, but is associated with large uncertainties due to poorly-known coupling constants of hyperons to meanfields, cf. [1,11]. The meson sector in (2) is treated on a mean-field level as described in Ref. [1].

The scaling parameters of the model, the "dielectric" constants $a_{i}$, renormalizations of the coupling constants and masses, $\tilde{\chi}_{i}$ and $\phi_{i}$, are dimensionless functions of the fields and effective couplings. The potential $\widetilde{U}(\sigma)$ represents the possible self-interaction of the $\sigma$ field, which was suggested in ref. [3]. In the general case the scaling parameters can depend also on $\left(\omega_{\mu} \omega^{\mu}\right)$ and $\left(\vec{\rho}_{\mu} \vec{\rho}^{\mu}\right)$ yielding corresponding terms in the potential. For instance, writing $\phi_{\omega}^{2}=\phi_{\omega}^{2}(\sigma)+\zeta \omega_{\mu} \omega^{\mu} / 4$ we recover the $\left(\omega_{\mu} \omega^{\mu}\right)^{2}$ terms proposed in ref. [30] to improve the description of finite nuclei within the RMF model approach. A systematic construction of mean-field Lagrangians with high-order terms based on constraints from the chiral symmetry of QCD can be found in ref. [31]. In our treatment we will neglect a possible dependence of the potential and the parameters on the $\omega$ and $\rho$ meson fields, elaborating only the $\sigma$-field depen- 
dence. We assume that the density dependent scaling functions $a_{N}$ and $\phi_{N}$ are basically due to a nucleon-sigma-meson interaction. Thus, we can write

$$
a_{N}=a_{N}\left(g_{\sigma} \tilde{\chi}_{\sigma} \sigma\right), \quad \phi_{N}=\phi_{N}\left(g_{\sigma} \tilde{\chi}_{\sigma} \sigma\right)
$$

Simplifying we will also assume that all other scaling functions, $\widetilde{\chi}_{\omega, \rho}, a_{\omega, \rho, \sigma}$, and the potential $\widetilde{U}$ are due to the same nucleon-sigma-meson interaction, i.e. are the functions of $g_{\sigma} \tilde{\chi}_{\sigma} \sigma$. For the rho meson included according to the hidden local symmetry principles [27] one has $g_{\rho}=g_{\rho}^{\prime}$. Despite this the scaling of $g_{\rho}$ and $g_{\rho}^{\prime}$ may be quite different in dense nuclear matter, $\tilde{\chi}_{\rho} \neq \tilde{\chi}_{\rho}^{\prime}$. The scaling factor $\tilde{\chi}_{\rho}$ arises due to the renormalization of the nucleon-sigma interaction in

medium, whereas the factor $\widetilde{\chi}_{\rho}^{\prime}$ is due to a non-Abelian interaction between $\rho_{i}^{\alpha}$ fields and does not depend directly on the nucleon-field source. Thus within above assumptions we may take $\widetilde{\chi}_{\rho}^{\prime} \simeq 1$.

The nucleon density is given by the zero component of the Noether current

$$
n_{N}=a_{N}<\Psi_{N}^{\dagger} \Psi_{N}>
$$

\subsection{Field redefinition in RMF models}

Describing an extended homogeneous system like a NS we may assume that mean fields of mesons do not depend on coordinates. Then the physical results obtained on a mean-field level would not change if all particle fields are rescaled by constant factors. For instance we may change meson and nucleon fields as

$$
\Psi_{N} \rightarrow \Psi_{N} / \sqrt{a_{N}}, \quad \sigma \rightarrow \sigma / \sqrt{a_{\sigma}}, \quad \omega_{\mu} \rightarrow \omega_{\mu} / \sqrt{a_{\omega}}, \quad \vec{\rho}_{\mu} \rightarrow \vec{\rho}_{\mu} / \sqrt{a_{\rho}}
$$

and obtain the new nucleon and meson Lagrangians equivalent to those in $(3,4)$ in a mean-field approximation

$$
\begin{aligned}
\mathcal{L}_{N}= & \bar{\Psi}_{N}(i D \cdot \gamma) \Psi_{N}-m_{N}^{*} \bar{\Psi}_{N} \Psi_{N}, \\
& D_{\mu}=\partial_{\mu}+i g_{\omega} \chi_{\omega} \omega_{\mu}+\frac{i}{2} g_{\rho} \chi_{\rho} \vec{\rho}_{\mu} \vec{\tau} \\
\mathcal{L}_{M}= & \frac{\partial^{\mu} \sigma \partial_{\mu} \sigma}{2}-\frac{m_{\sigma}^{* 2} \sigma^{2}}{2}-U(\sigma) \\
- & \frac{\omega_{\mu \nu} \omega^{\mu \nu}}{4}+\frac{m_{\omega}^{* 2} \omega_{\mu} \omega^{\mu}}{2}-\frac{\vec{\rho}_{\mu \nu} \vec{\rho}^{\mu \nu}}{4}+\frac{m_{\rho}^{* 2} \vec{\rho}_{\mu} \vec{\rho}^{\mu}}{2}, \\
& \omega_{\mu \nu}=\partial_{\nu} \omega_{\mu}-\partial_{\mu} \omega_{\nu}, \quad \vec{\rho}_{\mu \nu}=\partial_{\nu} \vec{\rho}_{\mu}-\partial_{\mu} \vec{\rho}_{\nu}+g_{\rho} \chi_{\rho}^{\prime}\left[\vec{\rho}_{\mu} \times \vec{\rho}_{\nu}\right] .
\end{aligned}
$$


We denoted $U(\sigma)=\widetilde{U}\left(\chi_{\sigma} \sigma\right)$. The effective masses of particles introduced in this Lagrangian are equal to

$$
\begin{aligned}
m_{N}^{*} / m_{N} & =\phi_{N}\left(\chi_{\sigma} \sigma\right) / a_{N}\left(\chi_{\sigma} \sigma\right)=\Phi_{N}\left(\chi_{\sigma} \sigma\right) \\
m_{i}^{*} / m_{i} & =\phi_{i}\left(\chi_{\sigma} \sigma\right) / \sqrt{a_{i}\left(\chi_{\sigma} \sigma\right)}=\Phi_{i}\left(\chi_{\sigma} \sigma\right), \quad i=\sigma, \omega, \rho,
\end{aligned}
$$

where the dimensionless functions $\Phi_{N}$ and $\Phi_{i}$ depend on the scalar field in the combination $\chi_{\sigma} \sigma$. The scaling functions of coupling constants $g_{\sigma}, g_{\omega}, g_{\rho}, g_{\rho}^{\prime}$ are given by

$$
\chi_{i}=\tilde{\chi}_{i}\left(\chi_{\sigma} \sigma\right) / \sqrt{a_{i}\left(\chi_{\sigma} \sigma\right)}, \quad i=\sigma, \omega, \rho, \quad \chi_{\rho}^{\prime}=\tilde{\chi}_{\rho}^{\prime}\left(\chi_{\sigma} \sigma\right) / \sqrt{a_{\rho}\left(\chi_{\sigma} \sigma\right)} .
$$

All quantities are now expressed in terms of the new renormalized $\sigma$ field.

The thermodynamic potential density $\Omega=-P$, where $P$ is the pressure, is related to the energy density by the standard equation

$$
E\left[n_{n}, n_{p}, n_{l} ; f, \vec{\rho}_{\mu}\right]=\sum_{i} \mu_{i} n_{i}+\Omega, \quad P=\sum_{i} \mu_{i} n_{i}-E, \quad \mu_{i}=\frac{\partial E}{\partial n_{i}}
$$

Chemical potentials enter the Green functions in the standard gauge combinations $\varepsilon_{i}+\mu_{i}$. Chemical potentials of the charged boson mean fields are also introduced by the gauge replacements $\varepsilon_{i} \rightarrow \varepsilon_{i}+\mu_{i}$.

For charged rho-meson fields above replacement implies

$$
\rho_{\mu \nu} \rightarrow \rho_{\mu \nu}+\Delta \rho_{\mu \nu}, \quad \Delta \rho_{\mu \nu}=\mu_{\mathrm{ch}}^{\rho} \delta_{\nu 0}\left[\overrightarrow{n_{3}} \times \overrightarrow{\rho_{\mu}}\right]-\mu_{\mathrm{ch}}^{\rho} \delta_{\mu 0}\left[\overrightarrow{n_{3}} \times \overrightarrow{\rho_{\nu}}\right]
$$

where $\left(\vec{n}_{3}\right)^{a}=\delta^{a 3}$ is the unit vector in the isospin space, $a=1,2,3$, and $\mu_{\mathrm{ch}}^{\rho}$ is the chemical potential of rho mesons. The explicit expression for $\Omega$ is given in Appendix.

After calculation of the nucleon contribution the energy-density functional takes the form

$$
\begin{aligned}
& E\left[n_{n}, n_{p}, n_{l} ; f, \omega_{0}, \vec{\rho}_{\mu}\right]=E_{N}\left[n_{n}, n_{p} ; f\right]+E_{l}\left[n_{e}, n_{\mu}\right] \\
& \quad+E_{\omega}\left[n_{n}, n_{p} ; f, \omega_{0}\right]+E_{\rho}\left[n_{n}, n_{p} ; f, \vec{\rho}_{\mu}\right], \\
& \begin{aligned}
E_{N}\left[n_{n}, n_{p} ; f\right]= & \frac{m_{N}^{4} f^{2}}{2 C_{\sigma}^{2}} \eta_{\sigma}(f)+U(f) \\
+ & \left(\int_{0}^{p_{\mathrm{F}, n}}+\int_{0}^{p_{\mathrm{F}, p}}\right) \frac{\mathrm{d} p p^{2}}{\pi^{2}} \sqrt{m_{N}^{2} \Phi_{N}^{2}(f)+p^{2}}
\end{aligned}
\end{aligned}
$$




$$
\begin{aligned}
& E_{l}\left[n_{e}, n_{\mu}\right]=\sum_{i=e, \mu} \int_{0}^{p_{\mathrm{F}, i}} \frac{\mathrm{d} p p^{2}}{\pi^{2}} \sqrt{m_{i}^{2}+p^{2}} \\
& \begin{aligned}
E_{\omega}\left[n_{n}, n_{p} ; f, \omega_{0}\right]= & \frac{C_{\omega}^{2}\left(n_{n}+n_{p}\right)^{2}}{2 m_{N}^{2} \eta_{\omega}(f)} \\
& -\frac{m_{N}^{2} \eta_{\omega}(f)}{2 C_{\omega}^{2}}\left[g_{\omega} \chi_{\omega} \omega_{0}-\frac{C_{\omega}^{2}\left(n_{p}+n_{n}\right)}{m_{N}^{2} \eta_{\omega}(f)}\right]^{2}, \\
E_{\rho}\left[n_{n}, n_{p} ; f, \vec{\rho}_{\mu}\right]=E_{\rho, \text { neut }}\left[n_{n}, n_{p} ; f, \rho_{0}^{(3)}\right]+E_{\rho, \mathrm{ch}}\left[n_{n}, n_{p} ; f, \vec{\rho}_{\mu}\right] & \\
E_{\rho, \text { neut }}\left[n_{n}, n_{p} ; f, \rho_{0}^{(3)}\right]= & \frac{C_{\rho}^{2}\left(n_{n}-n_{p}\right)^{2}}{8 m_{N}^{2} \eta_{\rho}(f)} \\
& -\frac{m_{N}^{2} \eta_{\rho}(f)}{2 C_{\rho}^{2}}\left[g_{\rho} \chi_{\rho} \rho_{0}^{(3)}-\frac{C_{\rho}^{2}\left(n_{p}-n_{n}\right)}{2 m_{N}^{2} \eta_{\rho}(f)}\right]^{2}, \\
E_{\rho, \text { ch }}\left[n_{n}, n_{p} ; f, \vec{\rho}_{\mu}\right]= & -\frac{m_{\rho}^{2}}{2} \Phi_{\rho}^{2}(f)\left(\vec{\rho}_{\mu} \vec{\rho}^{\mu}-\rho_{0}^{(3)} \rho_{0}^{(3)}\right) \\
& +\frac{1}{4}\left(\Delta \rho_{\mu \nu}\right)^{2}-\frac{1}{4} \mu_{\mathrm{ch}}^{\rho} \frac{\partial}{\partial \mu_{\mathrm{ch}}^{\rho}}\left(\Delta \rho_{\mu \nu}\right)^{2} .
\end{aligned}
\end{aligned}
$$

We have introduced here the dimensionless field

$$
f=g_{\sigma} \chi_{\sigma} \sigma / m_{N}
$$

and the dimensionless coupling constants $C_{i}=g_{i} m_{N} / m_{i}$, for $i=\sigma, \omega \rho$. The nucleon and lepton Fermi momenta $p_{\mathrm{F}, i}$ are related to the corresponding partial density as $n_{i}=p_{\mathrm{F}, i}^{3} /\left(3 \pi^{2}\right)$ for $i=n, p, e, \mu$. The contributions of mesonic fields entered with the scaling factors

$$
\eta_{i}(f)=\Phi_{i}^{2}(f) / \chi_{i}^{2}(f), \quad i=\sigma, \omega, \rho
$$

Then one may use equations of motion for $\omega_{0}$ and $\rho$-meson fields $\vec{\rho}_{\mu}$ :

$$
\begin{aligned}
& \frac{\partial}{\partial \omega_{0}} \Omega\left[n_{n}, n_{p} ; f, \omega_{0}\right]=\frac{\partial}{\partial \omega_{0}} E\left[n_{n}, n_{p} ; f, \omega_{0}\right]=0, \\
& \frac{\partial}{\partial \vec{\rho}_{\mu}} \Omega\left[n_{n}, n_{p} ; f, \vec{\rho}_{\mu}\right]=\frac{\partial}{\partial \vec{\rho}_{\mu}} E\left[n_{n}, n_{p} ; f, \vec{\rho}_{\mu}\right]=0 .
\end{aligned}
$$

The first line in (24) yields

$$
E_{\omega}\left[n_{n}, n_{p} ; f\right]=\frac{C_{\omega}^{2}\left(n_{n}+n_{p}\right)^{2}}{2 m_{N}^{2} \eta_{\omega}(f)}
$$


Traditionally one considers only a neutral $\rho$-meson field $\rho_{0}^{(3)}$ disregarding a possibility for the presence of charged $\rho$-meson mean fields. Then the second line in (24) yields

$$
\rho_{0}^{(3)}=g_{\rho} \chi_{\rho}\left(n_{p}-n_{n}\right) /\left(2 m_{\rho}^{2} \Phi_{\rho}^{2}\right)
$$

The corresponding contribution to the energy density is

$$
E_{\rho}\left[n_{n}, n_{p} ; f\right]=\frac{C_{\rho}^{2}\left(n_{n}-n_{p}\right)^{2}}{8 m_{N}^{2} \eta_{\rho}(f)}
$$

As one can see, the source of the isovector $\rho$-meson field $\rho_{0}^{(3)}$ is the isovector nucleon density $\left(n_{p}-n_{n}\right)$. Therefore for the isospin symmetrical matter $\left(n_{p}=\right.$ $n_{n}$ ) we always have $E_{\rho}=0$.

The main merit of this section is in the following. Eqs. (17-27) demonstrate that instead of twelve scaling functions of a scalar field, $a_{N, \sigma, \omega, \rho}, \tilde{\chi}_{\rho, \omega, \sigma}, \phi_{N, \sigma \omega, \rho}$ and $\widetilde{U}(\sigma)$, which enter the initial Lagrangian (2), the energy density functional depends actually only on four particular combinations of these functions, $\eta_{\sigma, \rho, \omega}(f)$ and $U(f)$. Note that the dependence on the scaling function $\eta_{\sigma}$ can be always presented as a part of the potential $U$ and absorbed in it by the replacement $U \rightarrow U+\frac{m_{N}^{4} f^{2}}{2 C_{\sigma}^{2}}\left(1-\eta_{\sigma}(f)\right)$, and vise versa the potential $U$ can be absorbed in $\eta_{\sigma}$. Thus only three independent functions enter the energy-density functional. The expression (17) demonstrates explicitly equivalence of mean-field Lagrangians for constant fields with various $a, \tilde{\chi}$ and $\phi$ parameters if they correspond to the same functions $\eta_{\rho, \omega}(f)$ and $\eta_{\sigma}$ (either $U(f))$. In sec. 3 we discuss possible constraints on the $f$ dependence of the functions. We would like to stress that the field $f$ relates now to a scalar field $\sigma$ in a non-trivial way determined by eq. (22).

In order to obtain final expression for the energy of the system, one should still use equation of motion for the scalar field $f$,

$$
\frac{\partial}{\partial f} \Omega\left[n_{n}, n_{p} ; f\right]=\frac{\partial}{\partial f} E\left[n_{n}, n_{p} ; f\right]=0
$$

\subsection{Determination of parameters of a RMF model}

Parameters of the RMF model, $C_{\sigma}, C_{\omega}, C_{\rho}$, and parameters of the potential $U$ are to be adjusted to reproduce the nuclear matter properties at the saturation and have rather broad uncertainties, cf. [32,33]. One usually takes the saturation density $n_{0} \simeq 0.16 \pm 0.015 \mathrm{fm}^{-3}$, the binding energy per particle, $e_{\mathrm{B}}\left(n_{0}\right) \simeq$ 
$-15.6 \pm 0.6 \mathrm{MeV}$, the compressibility modulus $K\left(n_{0}\right) \simeq 240 \pm 40 \mathrm{MeV}$, the effective nucleon mass $m_{N}^{*}\left(n_{0}\right) / m_{N} \simeq 0.75 \pm 0.1$, and the symmetry energy coefficient $a_{\text {sym }}\left(n_{0}\right)=32 \pm 4 \mathrm{MeV}$. Some models exploit parameters beyond even these large error bars. E.g., the original Walecka model [34] used $n_{0} \simeq 0.191 \mathrm{fm}^{-3}$ producing $K \simeq 540 \mathrm{MeV}$ and $m_{N}^{*} / m_{N} \simeq 0.56$.

To facilitate the comparison among different RMF models which we study below we will use the same basic input parameters

$$
\begin{aligned}
& n_{0}=0.16 \mathrm{fm}^{-3}, \quad e_{\mathrm{B}}=-16 \mathrm{MeV}, \quad a_{\mathrm{sym}}\left(n_{0}\right)=32 \mathrm{MeV} \\
& m_{N}=938 \mathrm{MeV}
\end{aligned}
$$

The saturation density and the binding energy are related as

$$
\left.\frac{\partial E[n ; f]}{\partial n}\right|_{n_{0}, f\left(n_{0}\right)}=\frac{1}{n_{0}} E\left[n_{0} ; f\left(n_{0}\right)\right]=m_{N}+e_{\mathrm{B}}
$$

and the compressibility modulus is given by

$$
K=9 n_{0}\left[\left.\frac{\partial^{2} E}{\partial n^{2}}\right|_{n_{0}, f\left(n_{0}\right)}-\left(\left.\frac{\partial^{2} E}{\partial n \partial f}\right|_{n_{0}, f\left(n_{0}\right)}\right)^{2}\left[\left.\frac{\partial^{2} E}{\partial f^{2}}\right|_{n_{0}, f\left(n_{0}\right)}\right]^{-1}\right]
$$

Here $f\left(n_{0}\right)$ is a solution of eq. (28) at the density $n_{p}=n_{n}=n_{0} / 2$. The coupling constant $C_{\rho}$ is determined from the symmetry energy coefficient of the nuclear matter

$$
a_{\mathrm{sym}}(n)=\left.\frac{n}{8} \frac{\partial^{2}}{\partial n_{p}^{2}} E\left[n-n_{p}, n_{p}\right]\right|_{n_{p}=n / 2}=\frac{C_{\rho}^{2} n}{8 m_{N}^{2} \eta_{\rho}}+\frac{\pi^{2} n}{4 p_{F} \sqrt{m_{N}^{* 2}+p_{\mathrm{FN}}^{2}}}
$$

$p_{\mathrm{FN}}$ is the nucleon Fermi momentum in isospin-symmetric matter.

Generic Lagrangians, $\mathcal{L}_{N}$ and $\mathcal{L}_{M}$ in (2) and the energy density functional (17) reproduce various types of mean-field models. Consider several examples. The choice

$$
\begin{aligned}
a_{N, \sigma, \omega, \rho} & =\widetilde{\chi}_{N, \sigma, \omega, \rho}=\phi_{\sigma, \omega, \rho}=1, \quad \phi_{N}=1-g_{\sigma} \sigma / m_{N}, \\
\widetilde{\chi}_{\rho}^{\prime} & =\widetilde{U}=0,
\end{aligned}
$$

yielding the energy-density functional $(17,18,25,27)$ with $\eta_{\sigma, \omega, \rho}=1$, and $\Phi_{N}=$ 
$1-f$, corresponds to the standard Walecka model $(\mathrm{W})^{3}$ with the minimal number of free parameters (since $U=0$ ) [34]. For (29) we find the parameter set

$$
\mathrm{W}: \quad C_{\sigma}^{2}=329.70, C_{\omega}^{2}=249.40, \quad C_{\rho}^{2}=68.09,
$$

which produces

$$
K \simeq 553 \mathrm{MeV}, \quad m_{N}^{*}\left(n_{0}\right) / m_{N} \simeq 0.54 .
$$

Thus this minimal model does not allow to fit appropriately the values of the nucleon effective mass and compressibility modulus. In order to cure these problems, it was suggested in ref. [3] to introduce a scalar-field self-interaction

$$
U(f)=m_{N}^{4}\left(b f^{3} / 3+c f^{4} / 4\right)=\frac{b}{3}\left(m_{N} g_{\sigma} \sigma\right)^{3}+\frac{c}{4}\left(g_{\sigma} \sigma\right)^{4} .
$$

Two additional parameters, $b$ and $c$, make the model (called the modified Walecka model $(\mathrm{MW})$ ) able to accommodate realistic values of the nuclear compressibility and effective nucleon mass. An extra attention should be paid to the fact that the coefficient " $c$ " should be positive, otherwise there is no stable vacuum for the Lagrangian $(4,11)^{4}$. The solid line in Fig. 1 shows values of $K$ and $m_{N}^{*}\left(n_{0}\right)$, for the boundary case $c=0$. Above this line $c>0$, and below $c<0$. With a positive $c$, low values of $m_{N}^{*}\left(n_{0}\right)$ correspond to unrealistically high values of the compressibility $K$.

Using as input (29) and

$$
K=270 \mathrm{MeV}, \quad m_{N}^{*}\left(n_{0}\right) / m_{N}=0.8
$$

we obtain

$$
\begin{array}{cl}
\mathrm{MW}: & C_{\sigma}^{2}=189.94, C_{\omega}^{2}=90.768, \quad C_{\rho}^{2}=100.18, \\
& b=6.3714 \times 10^{-3}, c=1.6288 \times 10^{-2} .
\end{array}
$$

The choice, cf. (2),

3 Inclusion of the neutral $\rho_{0}^{(3)}$ field generalizes the model to describe asymmetrical nuclear matter. We call the models, that include $\rho$-meson fields, by the same names as those without $\rho$-meson fields.

4 Some parameter sets used, e.g., in [1], do not respect this constraint dealing thus with a metastable state. One then assumes that a mean field energy is valid only near a local minimum. 


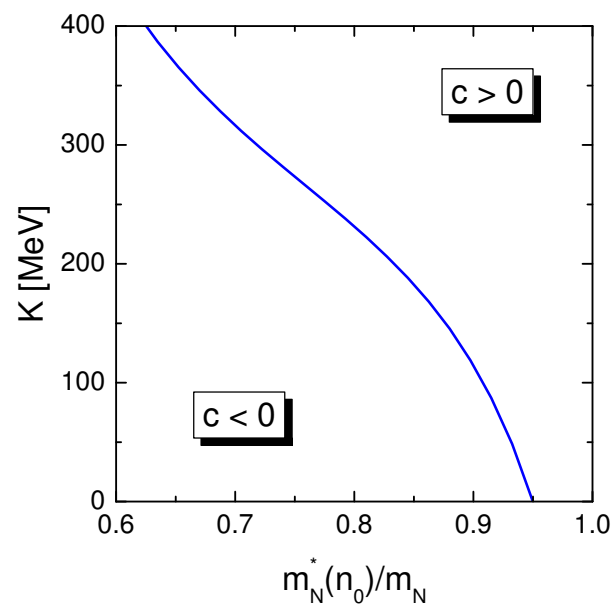

Fig. 1. The line shows the compressibility $K$ of the isospin symmetrical matter vs. the effective nucleon mass $m_{N}^{*}\left(n_{0}\right)$ corresponding to $c=0$ for the MW model, input set (29).

$$
\begin{aligned}
& a_{N}=1+g_{\sigma} \sigma / m_{N}, \quad a_{\sigma, \omega, \rho}=\phi_{N, \sigma, \omega, \rho}=\widetilde{\chi}_{N, \sigma, \omega, \rho}=1, \\
& \widetilde{\chi}_{\rho}^{\prime}=\widetilde{U}=0
\end{aligned}
$$

corresponds to the original Zimanyi-Moszkowski model with the derivative coupling (ZM) [36]. In eqs. $(17,18,25,27)$ we have then $U=0, \eta_{\sigma, \omega, \rho}=1$, $\Phi_{\sigma, \omega, \rho}=1$ and $\Phi_{N}=1 /(1+f)$. For the ZM model with the input parameters (29) we obtain

$$
\mathrm{ZM}: \quad C_{\sigma}^{2}=169.36, \quad C_{\omega}^{2}=59.055, \quad C_{\rho}^{2}=104.56
$$

This model reproduces realistic values of the compressibility modulus and the effective nucleon mass at $n_{0}$,

$$
K \simeq 224.5 \mathrm{MeV}, \quad m_{N}^{*}\left(n_{0}\right) / m_{N} \simeq 0.85
$$

without introduction of extra free parameters. As the model, which eliminates successfully the deficiencies of the original Walecka model keeping the minimal number of parameters, the ZM model has gained a lot of attention and has been widely used. Notice that, the ZM model can be also considered as a generalization of the $\mathrm{W}$ model done with the introduction of a scaling of the $\sigma N$ coupling

$$
g_{\sigma}^{*}=g_{\sigma} \chi_{\sigma}=g_{\sigma} /\left(1+g_{\sigma} \sigma / m_{N}\right) .
$$

To close this section we note that eqs. (13) and (14) demonstrate that for constant mean fields under consideration "dielectric constants" $a_{i}$ can be always 
absorbed into effective masses and effective coupling constants. Therefore, below we will put $a_{i}=1$ and deal with the functions $\Phi_{i}$ and $\chi_{i}$ only.

\section{RMF models with dropping hadron masses}

In this section we construct RMF models which support a decrease of hadron masses with an increase of the baryon density. Simplifying we follow the original Brown-Rho conjecture [19] about the hadron mass scaling, which in our case imposes

$$
\Phi_{N}=\Phi_{\sigma}=\Phi_{\omega}=\Phi_{\rho}=\Phi(f)
$$

Thus for the generalized W and MW models we would have $\Phi=\Phi_{\mathrm{W}}=$ $\Phi_{\mathrm{MW}}(f)=1-f$ and for the generalized ZM model, $\Phi=\Phi_{\mathrm{ZM}}(f)=1 /(1+f)$. We recall that $f$ is related to the $\sigma$ field via $(22)$.

We have to note that due to non-linear interactions effective masses obtained on a mean-field level should not be in general identified with positions of the poles in propagators of corresponding excitations. ${ }^{5}$ Following Brown and Rho [19] we apply a mass scaling on a mean-field level.

\subsection{RMF models with decreasing hadron masses and fixed coupling constants}

Assuming that coupling constants do not change in the medium, i.e. $\chi_{i}=1$, we obtain the following scaling factors in the energy-density functional

$$
\eta_{i}=\Phi^{2}(f), \quad i=\sigma, \omega, \rho
$$

For the generalized $\mathrm{W}$ and MW models we have $\Phi=\Phi_{\mathrm{W}}=\Phi_{\mathrm{MW}}(f)=1-f$ and for the generalized ZM model, $\Phi=\Phi_{\mathrm{ZM}}(f)=1 /(1+f)$. In terms of a $\sigma$ field these functions have familiar forms $\Phi_{\mathrm{MW}}(\sigma)=1-g_{\sigma} \sigma / m_{N}$ and $\Phi_{\mathrm{ZM}}(\sigma)=1 /\left(1+g_{\sigma} \sigma / m_{N}\right)$.

The resulting functional possesses several unpleasant features. First, effective hadron masses do not decrease monotonously with the density increase. Decreasing at small densities, they start to increase at higher densities. Such a non-linear behavior has been observed, e.g., in ref. [22]. To show that this is a generic feature of such a type of models we calculate $\partial f(n) / \partial n$, where $f(n)$

$\overline{5}$ In our case a difference appears for the $\sigma$-meson and nucleon masses. 
is a solution of eq. (28) for isospin symmetrical nuclear matter with the total density $n=n_{p}+n_{n}$. From (28) we have

$$
\frac{\partial f}{\partial n}=-\left.\frac{\partial^{2} E_{N}[n ; f]}{\partial n \partial f}\right|_{f(n)} /\left.\frac{\partial^{2} E_{N}[n ; f]}{\partial f^{2}}\right|_{f(n)}
$$

where the denominator $\partial^{2} E_{N} / \partial f^{2}$ should be always positive to assure the stability of the mean-field solution (28). The nominator,

$$
\left.\frac{\partial^{2} E[n ; f]}{\partial n \partial f}\right|_{f(n)}=-\frac{C_{\omega}^{2} n}{m_{N}^{2} \eta_{\mathrm{V}}^{2}} \frac{\partial \eta_{\omega}}{\partial f}+\frac{\partial \Phi_{N}}{\partial f} \frac{m_{N}^{2} \Phi_{N}(f)}{\sqrt{m_{N}^{2} \Phi_{N}^{2}(f)+p_{\mathrm{F}}^{2}}}
$$

changes the sign at some value of $n$, provided $\partial \eta_{\omega} / \partial f$ and $\partial \Phi_{N} / \partial f$ have the same sign. Indeed at small densities the second term dominates, whereas with the density increase the first term begins to win. Thus, if $f$ increases at small densities (hadron masses drop), at larger densities $f$ begins to decrease, resulting in an increase of hadronic masses. This effect can be enhanced in the isospin asymmetric nuclear matter due to the rho-meson contribution, if $\partial \eta_{\rho} / \partial f$ has the same sign as $\partial \eta_{\omega} / \partial f$ and $\partial \Phi_{N} / \partial f$. The second feature of the energy-density functional (17) with scaling factors (44) is that it does not guarantee the continuity of the density dependence of the scalar field $f$. The equation of motion (28) for $f$ may have several solutions, which can appear or disappear with a change of the density, leading to jumps of the $f(n)$ function. Third, the scaling of the mass of the scalar $\sigma$-meson field included in a DiracBrueckner-Hartree-Fock calculation of nuclear matter leads to over-binding of the nuclear matter, as has been found in ref. [37]. Fourth, in ref. [38] it was noticed that increasing the vector repulsive potential, due to the factor $1 / \eta_{\omega}$, would produce too strong nucleon flow in heavy-ion collisions. Such a flow would be in odd with experimental data. This observation stimulated authors of ref. [20] to propose the scaling of the vector-meson coupling constant $\chi_{\omega} \sim \Phi_{\omega}$, which leads to $\eta_{\omega} \simeq 1$. In the scalar sector the problems can be solved if we put $\eta_{\sigma} \simeq 1$ and choose the potential $U$ so that there is only one solution of the equation of motion for $f$.

Information about density dependence of the symmetry energy $a_{\text {sym }}(n)$, cf. (32), is rather controversial. The microscopic calculation within the relativistic Dirac-Brueckner-Hartree-Fock approach [35] shows that $a_{\text {sym }}(n)$ increases almost linearly with the density. This would require $\eta_{\rho} \simeq 1$ in (32). Ref. [25] discussing heavy ion collision properties uses a stiffer density dependence of $a_{\text {sym }}(n)\left(\eta_{\rho}\right.$ decreases with a density increase). On an other hand, the HHJ EoS [16] fitting the microscopic model of Urbana-Argonne $\left(\mathrm{A} 18+\delta v+\mathrm{UIX}^{*}\right)[2]$ produces $a_{\text {sym }}(n) \propto n^{0.6}$ that corresponds to increasing $\eta_{\rho} \simeq\left(n / n_{0}\right)^{0.4}$. Note that the latter case allows to solve the problem of the low DU threshold density. 


\subsection{RMF models with a universal scaling of hadron masses and coupling constants}

\subsubsection{Construction of the model}

Following the conjecture of ref. [20] and argumentation in the previous subsection we study now the case of a universal scaling of hadron masses and coupling constants,

$$
\Phi_{N}=\Phi_{\sigma}=\Phi_{\omega}=\Phi_{\rho}=\chi_{\sigma}=\chi_{\omega}=\chi_{\rho}=\Phi(f),
$$

which implies

$$
\eta_{\sigma}=\eta_{\omega}=\eta_{\rho}=1
$$

Note that according to a hypothesis of a "vector manifestation" [39] the vector meson mass must go to zero in proportion to the corresponding coupling. At the saturation nuclear matter density refs. $[20,40]$ give $\Phi\left(n_{0}\right) \simeq 0.78$ according to their analysis of the gyro-magnetic ratio that agrees with our input value $(37), m_{N}^{*}\left(n_{0}\right) / m_{N} \simeq 0.8$.

Note also that for the case $\chi_{\sigma} \neq 1$ we have a non-trivial relation between $f$ field and an original $\sigma$ field (22). The $\mathrm{W}$ and ZM models (in both of them $U=0)$ with the universal scaling $(47,48)$ we will denote as the $\mathrm{W}(\mathrm{u})$ and $\mathrm{ZM}(\mathrm{u})$ models, respectively, and the MW model with the universal scaling we will denote $\mathrm{MW}(\mathrm{u})$. For the $\mathrm{W}(\mathrm{u})$ and $\mathrm{MW}(\mathrm{u})$ models we then have

$$
\Phi(f)=1-f \quad \longrightarrow \quad f=\frac{g_{\sigma} \sigma / m_{N}}{1+\frac{g_{\sigma} \sigma}{m_{N}}} \quad \longrightarrow \quad \Phi(\sigma)=\frac{1}{1+\frac{g_{\sigma} \sigma}{m_{N}}} .
$$

For the $\mathrm{ZM}(\mathrm{u})$ model the correspondence is a more complex:

$$
\begin{aligned}
\Phi(f)=(1+f)^{-1} & \longrightarrow f=\sqrt{\frac{1}{4}+\frac{g_{\sigma} \sigma}{m_{N}}}-\frac{1}{2} \\
& \longrightarrow \Phi(\sigma)=\left(\sqrt{\frac{1}{4}+\frac{g_{\sigma} \sigma}{m_{N}}}+\frac{1}{2}\right)^{-1} .
\end{aligned}
$$

We note the obvious equivalence between the original Walecka model (W) and the $\mathrm{W}(\mathrm{u})$ model, and also between the original $\mathrm{ZM}$ and $\mathrm{ZM}(\mathrm{u})$ models for constant mean fields. We will denote models with a non-universal scaling as (nu) models. From (49) we see that W(u) model is in turn equivalent to 
the $\mathrm{ZM}(\mathrm{nu})$ model with $\Phi_{\sigma}(\sigma)=\Phi_{N}(\sigma)=1 /\left(1+g_{\sigma} \sigma / m_{N}\right)$ but $\chi_{\sigma}=1$ and $\Phi_{\omega, \rho}=\chi_{\omega, \rho}=1$. Analogously we find equivalence of the $\mathrm{ZM}(\mathrm{u})$ model and the $\mathrm{W}(\mathrm{nu})$ model with $\Phi_{\sigma, \omega, \rho}=\chi_{\omega, \rho}=1$ and $\chi_{\sigma}(\sigma)=\Phi_{N}(\sigma)=1-g_{\sigma} \sigma / m_{N}$.

As it follows from $(10,11,12,13,14)$, and (47) in the absence of the charged $\rho$ meson mean fields and the potential $U$, with the help of the replacements

$$
\Phi \xi \rightarrow \xi, \quad \text { where } \quad \xi=\sigma, \omega, \rho
$$

we can equivalently transform a RMF model with a universal scaling of meson masses and coupling constants $\left(\eta_{\sigma, \omega, \rho}=1\right)$ to a model without any scaling of meson masses and coupling constants. The absence of the non-Abelian interaction of $\rho$ meson fields and the potential $U$ is very important in order to arrive at such a conclusion, since the transition (51) would affect the non-linear sectors of the model.

For the MW model with $U$ given by (36) we can construct an equivalent MW(u) model with the universal scaling of meson masses and coupling constants (47) for $\Phi=1-f$ and the same form of $U$ written in terms of an $f$ field. In terms of the $\sigma$ field the potential of the new MW(u) model looks more cumbersome:

$$
U=m_{N}^{4}\left(\frac{b}{3} f^{3}+\frac{c}{4} f^{4}\right)=\frac{b}{3} \frac{m_{N}\left(g_{\sigma} \sigma\right)^{3}}{\left(1+\frac{g_{\sigma} \sigma}{m_{N}}\right)^{3}}+\frac{c}{4} \frac{\left(g_{\sigma} \sigma\right)^{4}}{\left(1+\frac{g_{\sigma} \sigma}{m_{N}}\right)^{4}} .
$$

Note that potentials $U(\sigma)$ in the MW and MW(u) models, cf. $(36,52)$, look quite differently in terms of the $\sigma$ field although in terms of the $f$ fields they are the same. We used the same letter $f$, since the mean values of those $f$ fields, determined from the very same equation of motion, are identical. This illustrates that, if thermodynamic characteristics look the same in terms of the variables found in the minimization procedure, then the RMF models are equivalent.

As an illustration we also exploit a simplified ansatz proposed in ref. [20] (further the SBMR model) for the isospin symmetrical matter, where the Brown-Rho scaling was included into RMF EoS. Assume that the energy density of the model takes the form that we have used above with

$$
\begin{aligned}
& \eta_{\omega}=\Phi^{2}(n ; z) / \Phi^{2}(n ; y), \quad \eta_{\sigma}=\Phi^{2}(n ; y), \quad \Phi_{N}(n, f)=\Phi(n ; y)-f \\
& \Phi(n ; y)=1 /\left(1+y p_{\mathrm{F}}^{3}(n) /(260 \mathrm{MeV})^{3}\right) \quad p_{\mathrm{F}}^{3}(n)=\left(p_{\mathrm{F}, i}^{3}+p_{\mathrm{F}, i}^{3}\right) / 2 .
\end{aligned}
$$

Here $f=h \sigma / m_{N}, h$ is the coupling constant and $\sigma=\phi$ in notations of [20], eq. (32). Parameters of the model are $m_{N}=939 \mathrm{MeV}, y=0.28, z=$ 

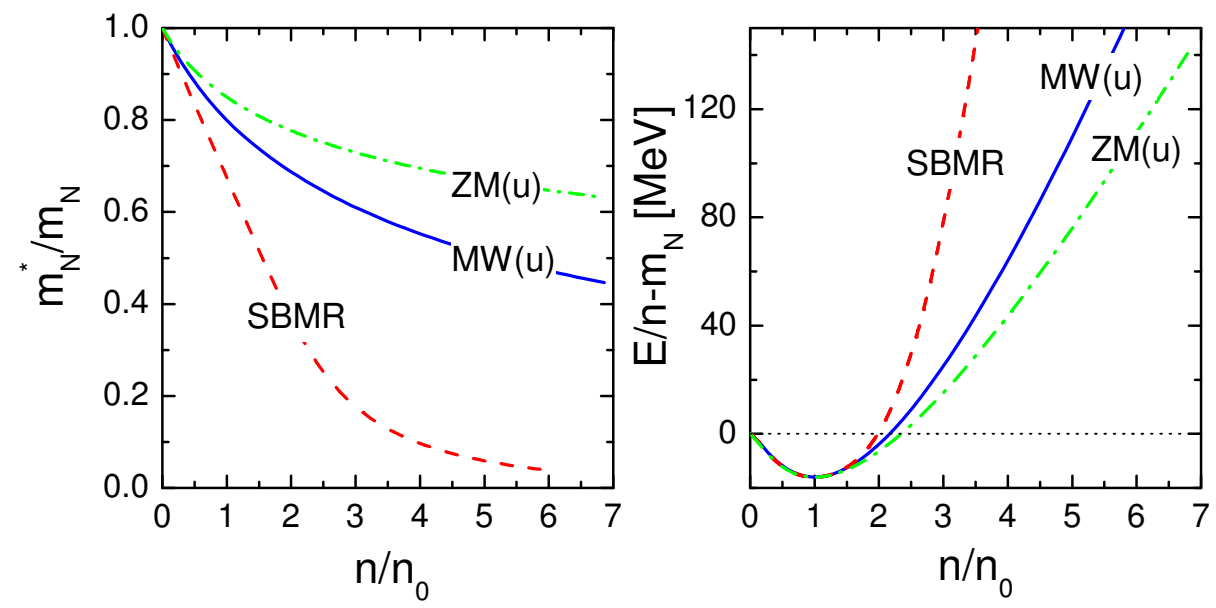

Fig. 2. The effective nucleon mass (left panel) and the energy per particle (right panel) of the isospin symmetrical nuclear matter within the MW(u) (input-parameter set $(29,37,38)), \mathrm{ZM}(\mathrm{u})$ (input-parameter set $(29,40))$ and SBMR model (53).

0.31, $C_{\omega}^{2}=332.27$ and $C_{\sigma}^{2}=50.546$, which correspond to $n_{0}=0.151 \mathrm{fm}^{-3}$, $e_{B}=-16.1 \mathrm{MeV}, K=259.6 \mathrm{MeV}$, and $m_{N}^{*}\left(n_{0}\right)=0.675 m_{N}$. Here $\eta_{\omega}$ is very closed to unit and $\eta_{\sigma}$ depends on $n$ rather than on $f$. The pressure and chemical potentials of partilces are related to the energy following eqs. (15). Note that owing to an explicit dependence of the energy on the nucleon density expressions for the neutron and proton chemical potentials deviate from those given by eqs. (A.10) and (A.11).

In order to consider asymmetrical matter within the same framework we add the rho-meson contribution (27) with $\eta_{\rho}=1$ and $C_{\rho}^{2}=100.08$. The latter quantity follows from the value of $a_{\mathrm{sym}}\left(n_{0}\right)=32 \mathrm{MeV}$ in (29).

In Fig. 2 we show the effective nucleon mass and the EoS for the isospin symmetrical nuclear matter calculated for three models of EoS: MW(u) (inputparameter set $(29,37,38)), \mathrm{ZM}(\mathrm{u})$ (input-parameter set $(29,40)$ ) and SBMR, (53). We see that these three models cover a broad range of possible density dependences of the effective mass and the energy per particle. Compared to the RMF models the SBMR model produces very rapid decrease of the effective nucleon mass and a stiff EoS (at a reasonable value of the compressibility for $\left.n=n_{0}\right)$.

\subsubsection{Neutron star composition}

The composition of the NS matter (in absence of mixed phases and a charged condensate) is determined in the standard manner by the local charge-neutrality condition and the conditions of the equilibrium with respect to weak reactions $e \leftrightarrow \mu$ and $n \leftrightarrow p+e$. The latter ones impose chemical equilibrium conditions 

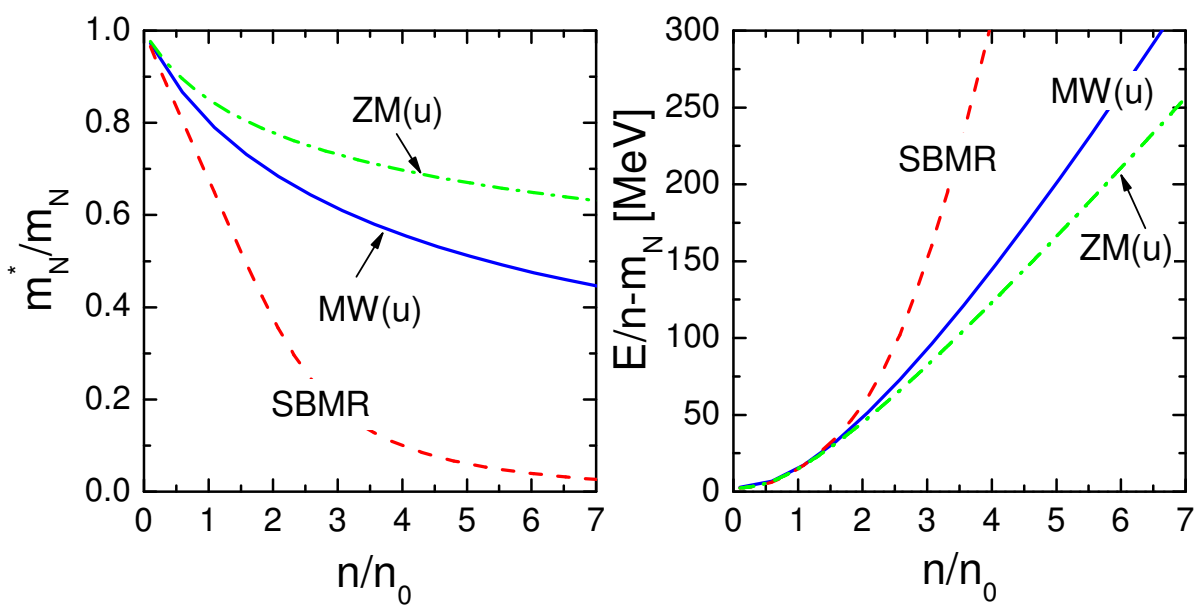

Fig. 3. The same as in Fig. 2 but for the neutron star matter.

for the corresponding chemical potentials

$$
\mu_{e}=\mu_{\mu}=\mu_{n}-\mu_{p}, \quad \mu_{i}=\frac{\partial E_{\mathrm{tot}}}{\partial n_{i}}, \quad i=e, \mu, n, p
$$

Here $E_{\text {tot }}$ is the full energy-density. For the energy density (17) conditions (54) and the local charge-neutrality condition result in the system of equations

$$
\begin{aligned}
& \mu_{e}=\sqrt{m_{N}^{2} \Phi_{N}^{2}(\bar{f})+p_{\mathrm{F}, n}^{2}}-\sqrt{m_{N}^{2} \Phi_{N}^{2}(\bar{f})+p_{\mathrm{F}, p}^{2}}+\frac{C_{\rho}^{2}\left(n_{n}-n_{p}\right)}{2 m_{N}^{2} \eta_{\rho}(\bar{f})} \\
& n_{p}=\frac{\left(\mu_{e}^{2}-m_{e}^{2}\right)^{\frac{3}{2}}}{3 \pi^{2}} \theta\left(\mu_{e}^{2}-m_{e}^{2}\right)+\frac{\left(\mu_{e}^{2}-m_{\mu}^{2}\right)^{\frac{3}{2}}}{3 \pi^{2}} \theta\left(\mu_{e}^{2}-m_{\mu}^{2}\right)
\end{aligned}
$$

with $\bar{f}=f(n)$ being a solution of the equation of motion (28) for the scalar field. Eqs. (55) are solved with respect to $n_{p}$ for a given total density $n=$ $n_{p}+n_{p}$. In Fig. 3 we show the effective nucleon mass and the EoS for the NS matter calculated for MW(u) (input-parameter set $(29,37,38)$ ), ZM(u) (input-parameter set $(29,40)$, and SBMR models. Compared to the isospin symmetrical case (Fig. 2) the EoS of the NS matter becomes substantially stiffer for all three models.

In Fig. 4 (left and middle panels) we show the composition of the NS matter and the electron chemical potential, as they follow from the solution of equations (54), (55) for the three specified above models (MW(u), ZM(u), and SBMR). We see that all three models produce the very similar NS compositions and $\mu_{e}$. The proton concentration $Y_{p}=n_{p} / n$ is rather high so that the DU process becomes operative at $n=2.13 n_{0}, 2.07 n_{0}$, and $1.72 n_{0}$ for the $\mathrm{ZM}(\mathrm{u}), \mathrm{MW}(\mathrm{u})$, and SBMR models, respectively. The corresponding NS masses are $M=0.88,0.99$, and $0.97 M_{\odot}$. Thus, yielding so low DU threshold densities these models cannot adequately describe the NS cooling, cf. [10]. This 

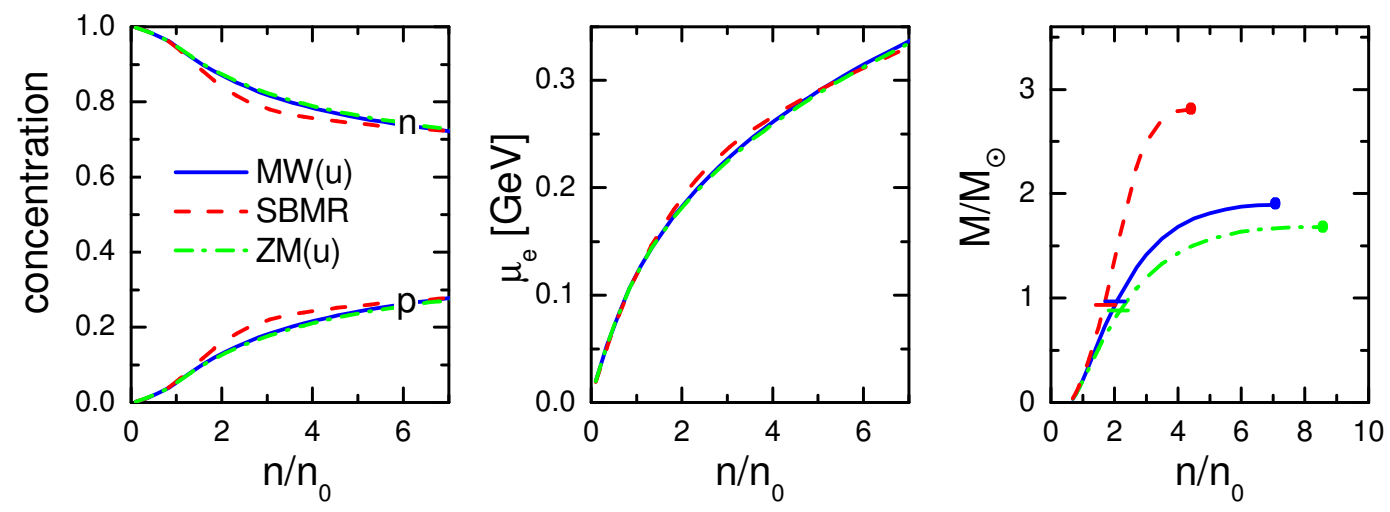

Fig. 4. The proton and neutron concentrations (left panel), and the electron chemical potential (middle panel) in the neutron star matter calculated for the same models as in Figs 2, 3. The right panel shows the neutron star mass as a function of the central density. Horizontal cuts indicate the DU thresholds.

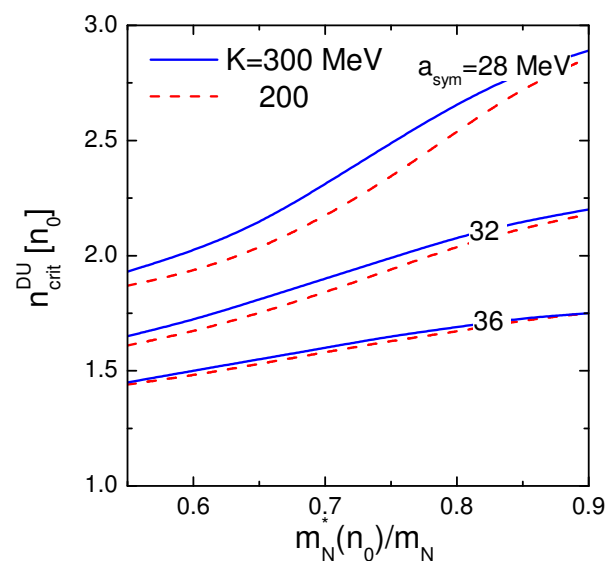

Fig. 5. The threshold density for the DU process as a function of the $m_{N}^{*}\left(n_{0}\right)$ for the neutron star matter, for the different MW(u) models, the input set (29), at two values of $K$ and three values $a_{\text {sym }}$.

is a serious drawback. In the right panel of Fig. 4 we show the NS masses (in units of solar mass $\left.M_{\odot}=2 \times 10^{33} \mathrm{~g}\right)$ resulting from the integration of the differential Oppenheimer-Volkoff equations [1] for a given central density of the star. We stopped the integration at the inner crust-core boundary, because the EoS following (17) cannot be used any longer. In different models the value of this boundary density varies in the interval $n_{\text {crust }} \simeq(0.5 \div 0.8) n_{0}$. In our calculations we settle $n_{\text {crust }} \simeq 0.8 n_{0}$. Since the crust contributes only a little to the NS mass (yielding an additional mass $\leq 0.06 M_{\odot}$ ), we do not supplement our calculations by subsequent calculation of the crust. We see that the $\mathrm{ZM}(\mathrm{u})$ RMF model has an extra shortcoming that the limiting NS mass is rather low. Opposite, the SBMR model shows an example of a very stiff $\operatorname{EoS}\left(M_{\lim } \simeq 2.8 M_{\odot}\right)$.

It is interesting to study the dependence of a DU threshold density and a limiting NS mass on the input parameters of a RMF model. We will use 


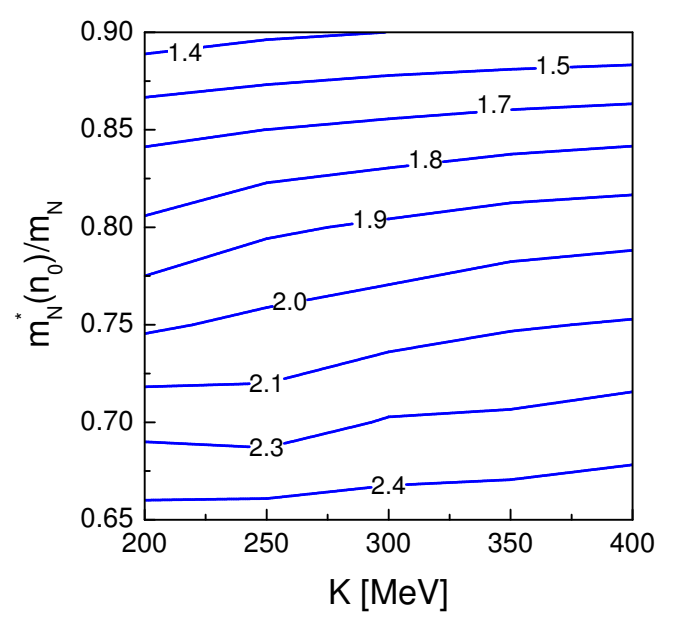

Fig. 6. The counter plot of the limiting masses of neutron stars (counter labels in $\left.M_{\odot}\right)$, for the MW(u) models, the input set (29), for different values of the input parameters $K$ and $m_{N}^{*}\left(n_{0}\right)$.

the MW(u) keeping the basic input parameters (29) and varying parameters $m_{N}^{*}\left(n_{0}\right)$ and $K$. Fig. 5 shows threshold densities for the DU process as a function of the magnitude of the effective nucleon mass at $n_{0}$ for two values of the compressibility $K=200$ and $300 \mathrm{MeV}$ and for three values of the symmetry energy coefficient $a_{\text {sym }}\left(n_{0}\right)=28,32,36 \mathrm{MeV}$. We see that $n_{\text {crit }}^{\text {DU }}$ increases with increase of $K$, and $m_{N}^{*}\left(n_{0}\right)$ and decreases with increase of $a_{\text {sym }}$. In all cases $n_{\text {crit }}^{\mathrm{DU}}$ is more sensitive to the changes of $a_{\text {sym }}$ and $m_{N}^{*}\left(n_{0}\right)$, than to the change of the compressibility. We see that all RMF models yield $n_{\text {crit }}^{\mathrm{DU}}<$ $2.7 n_{0}$ for reasonable values of parameters. Hence all these models will meet difficulties in applications to a NS cooling problem, cf. NS masses in Fig. 4. As we have mentioned, models with $M\left(n_{\text {crit }}^{\mathrm{DU}}\right)<1.3 M_{\odot}$ meet serious problems, since the latter value is smaller than the averaged value of the NS mass, $M \simeq 1.35 \pm 0.04 M_{\odot}$, and models producing $M\left(n_{\text {crit }}^{\text {DU }}\right)<1 M_{\odot}$ should be completely excluded.

The dependence of the limiting NS mass on the input parameters is illustrated in Fig. 6. The limiting mass is a sharp function of $m_{N}^{*}\left(n_{0}\right)$ and a rather smooth function of $K$. We see that the limiting mass $>(2.2 \div 2.3) M_{\odot}$ can be reached either for models exploiting unrealistic values of the compressibility modulus $K>300 \mathrm{MeV}$, or for $m_{N}^{*}\left(n_{0}\right)<0.7 m_{N}$. Therefore if one observed a NS of a mass $M \gtrsim 2.2 M_{\odot}$, see [12], this would mean that many RMF models without any scaling or with a universal scaling of masses and coupling constants would have a problem. One of the possibility to avoid the problem is to use $m_{N}^{*}\left(n_{0}\right)<0.7 m_{N}$. However, in the latter case one may meet a problem with description of the atomic nucleus data. Besides then either the compressibility is unrealistically high or $c<0$, as it follows from Fig. 1. 


\subsection{RMF models with a non-universal scaling for masses and couplings}

\subsubsection{Solving problems with DU reactions and limiting NS mass}

Obviously the universal scaling law may hold only approximately. In this section we demonstrate that allowing some departure from the universal scaling of the hadron masses and the coupling constants (47) we are able to obtain a somewhat stiffer EoS and shift the threshold density for the DU process to higher densities. We will consider the MW(nu) models with $\eta_{\sigma}=1$ but $\eta_{\omega}(f)<1$, being decreasing with $n$ for $n>n_{0}$. This behavior enhances the repulsion in the potential at large densities. In absence of negatively charged bosons to increase the critical density for the DU processes we should reduce the proton concentration. This can be achieved by choosing $\eta_{\rho}(f)>1$, being increasing with $n$ for $n>n_{0}$, that results in a suppression of the rho-meson contribution to the EoS.

As we have argued in section 3.1, if $\eta_{\omega}^{\prime}(f)<0$, then a decrease of a hadron mass will stop at some density and turn to an increase. However we can choose the scaling to be weak enough to shift a turning point to a high density not reached in the center of the heaviest NS (of the limiting mass). In the case of the NS matter we can exploit the fact that the first term in (46) responsible for the turn-over of the hadron mass scaling can be partially compensated by the analogous term from the rho-meson contribution, if $\eta_{\rho}^{\prime}(f)>0$. For the pure neutron matter the exact cancellation condition takes the form $4 C_{\omega}^{2} \eta_{\omega}^{\prime}(f) \eta_{\rho}^{2}(f)=-C_{\rho}^{2} \eta_{\rho}^{\prime}(f) \eta_{\omega}^{2}(f)$. An appropriate behavior of the scaling factors is obtained, e.g., with

$$
\eta_{\sigma}=1, \quad \eta_{\omega}(f)=\frac{1+z f_{0}}{1+z f}, \quad \eta_{\rho}(f)=\frac{\eta_{\omega}(f)}{\eta_{\omega}(f)+4 \frac{C_{\omega}^{2}}{C_{\rho}^{2}}\left(\eta_{\omega}(f)-1\right)}
$$

where $f_{0}=1-m_{N}^{*}\left(n_{0}\right) / m_{N}$. Since $\eta_{\omega}\left(f_{0}\right)=\eta_{\rho}\left(f_{0}\right)=1$, the values of parameters $C_{\omega}$ and $C_{\rho}$ do not change. Other parameters related to the scalar field change just slightly.

We fit now the parameter $z$ in order to increase the limiting NS mass $M_{\lim }$ and to push the threshold density $n_{\text {crit }}^{\text {DU }}$ to higher densities. The optimal value is found to be $z=0.65$. We use the same parameter set (29), as before. Had we taken the same values of the compressibility modulus $K=270 \mathrm{MeV}$ and $m_{N}^{*}\left(n_{0}\right) / m_{N}=0.8$, as we have used above, we would obtain negative value of $c$. The " $c=0 "$ border shown in Fig. 1 shifts slightly up, if we apply the scaling (56). In order to get positive $c$ we take slightly different values for the effective mass and the compressibility modulus compared to those in (38). We use 


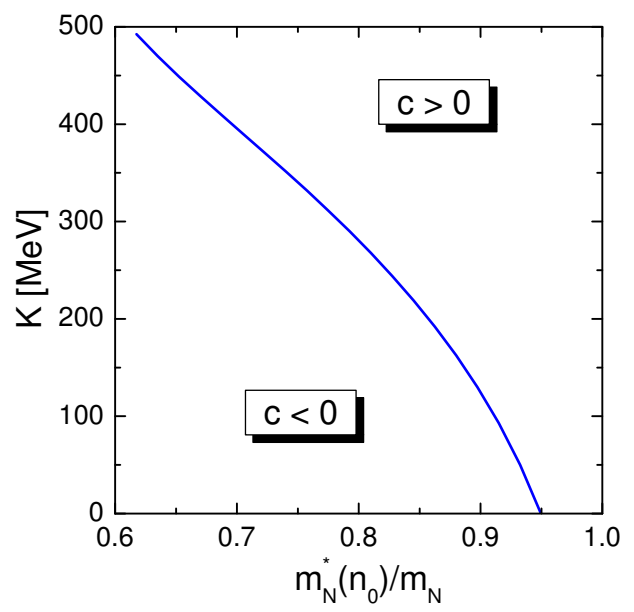

Fig. 7. The line shows the compressibility $K$ of the isospin symmetrical nuclear matter vs. the effective nucleon mass $m_{N}^{*}\left(n_{0}\right)$ corresponding to $c=0$ for the $\mathrm{MW}(\mathrm{nu})$ model with the scaling (56) and $z=0.65$ for the input set (29).

$$
m_{N}^{*}\left(n_{0}\right) / m_{N}=0.805, \quad K=275 \mathrm{MeV} .
$$

Then we obtain

$$
\begin{array}{ll}
\text { for } \mathrm{MW}(\mathrm{nu}) \quad & z=0.65: \\
& C_{\omega}^{2}=87.600, \quad C_{\rho}^{2}=100.64, \quad C_{\sigma}^{2}=179.56, \\
& b=7.7346 \times 10^{-3}, \quad c=3.4462 \times 10^{-4}
\end{array}
$$

and

$$
\begin{array}{ll}
\text { for } \mathrm{MW}(\mathrm{u}) \quad & z=0 \text { : } \\
& C_{\omega}^{2}=87.600, \quad C_{\rho}^{2}=100.64, \quad C_{\sigma}^{2}=184.36 \\
& b=5.5387 \times 10^{-3}, \quad c=2.2976 \times 10^{-2}
\end{array}
$$

In Fig. 7 we show a new $c=0$ border on the $\left(K, m_{N}^{*}\right)$-plane for the MW(nu) models using the input set (29) and the scaling functions (56) for $z=0.65$. We draw the same conclusion as in the case of MW(u) models. In the framework of MW(nu) models (with $z=0.65$ ) keeping $c>0$ we can't significantly decrease the effective nucleon mass $m_{N}^{*}\left(n_{0}\right)$ not increasing the compressibility beyond realistic values.

A very small value of the $c$-parameter in (58) that we have obtained, motivates a conjecture that the number of free parameters of the model can be still reduced. If we did not care about the global stability of the ground state we would put $c=0$ in (58). Taking care of this problem we could construct a model assuming $b=0$, but $c>0$. Exploring $\eta_{\sigma} \neq 1$ we could put $b=c=0$. 

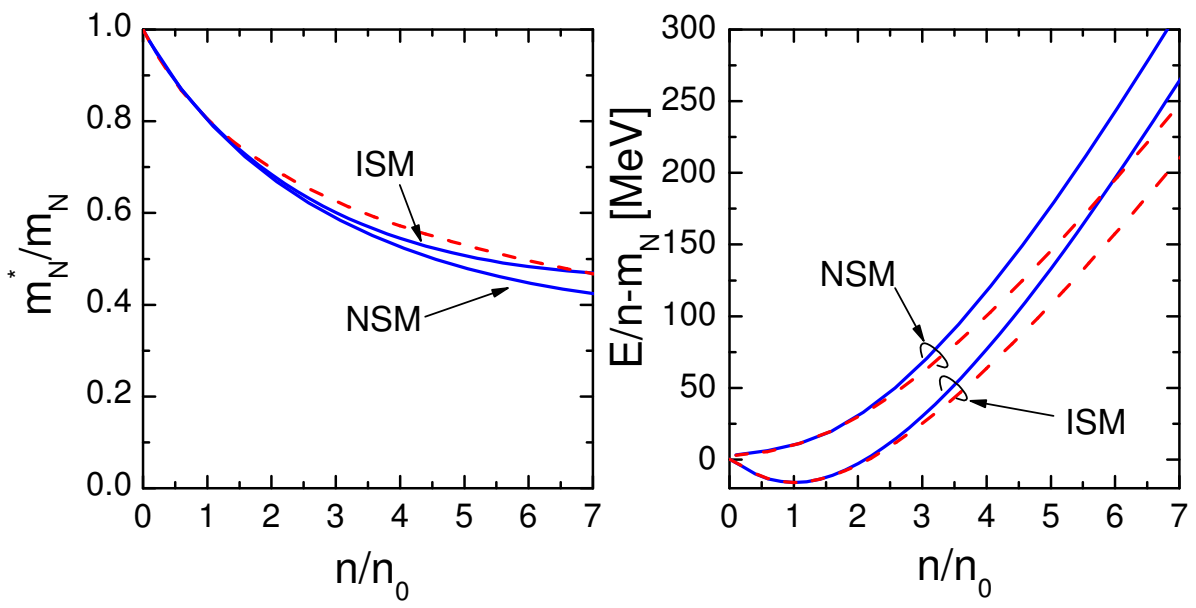

Fig. 8. The effective nucleon mass (left panel), and the energy per particle (right panel) of the isospin symmetrical nuclear matter (ISM) and the neutron star matter (NSM) within the MW(nu) model with the scaling (56), $z=0.65$, input-parameter set $(29,57,58)$. The dashed lines correspond to the MW(u) model $(z=0)$ with the input-parameter set $(29,57,59)$.

In this paper we will not consider these possibilities of further modernizations of our models.

Fig. 8 (left) demonstrates the density dependence of the effective nucleon mass for the isospin symmetrical nuclear matter (ISM) and for the NS matter (NSM) for the given MW(nu) model (solid lines: $z=0.65$, see $(29,56,57,58)$ ), and for the MW(u) model (dashed lines: $z=0,(29,56,57,59)$ ). Dashed curves for ISM and NSM cases cannot be distinguished. We find that masses are slightly smaller for the MW(nu) model. We observe that for $z=0.65$ the effective nucleon mass is monotonously decreasing in the symmetrical nuclear matter up to the density of $7 n_{0}$. The effective nucleon mass becomes to increase only at higher densities. A dis-balance of the universal scaling is just minor. Right panel in Fig. 8 shows energies per particle. They are higher in case of the MW(nu) model (for $n \gtrsim 2 n_{0}$ ).

In Fig. 9 (left) we show how the given MW(nu) model (the input-parameter set $(29,56,57,58))$, may help to cure a problem of the low critical density for the DU process. For the MW(nu) model the DU process becomes forbidden up to $4.0 n_{0}$, whereas for the given $\mathrm{MW}(\mathrm{u})$ model (the input-parameter set $(29,56,57,59))$ we have $n_{\text {crit }}^{\mathrm{DU}} \simeq 2.08 n_{0}$. The middle panel demonstrates the behavior of the electron chemical potential. The right panel shows the NS mass as a function of central density. With the $M W(n u)$ model $(z=0.65)$ the limiting NS mass is increased by about $5 \%$. We see that due to a stiffening of the EoS we may now satisfy the mentioned limit $M \gtrsim 2 M_{\odot}$. If we used another parameter choice, e.g., with a smaller effective nucleon mass at $n_{0}$, we could construct an even stiffer EoS. The critical value of the NS mass for the occurrence of the DU process is $M\left(n_{\text {crit }}^{\mathrm{DU}} \simeq 4.0 n_{0}\right) \simeq 1.69 M_{\odot}$. This value 

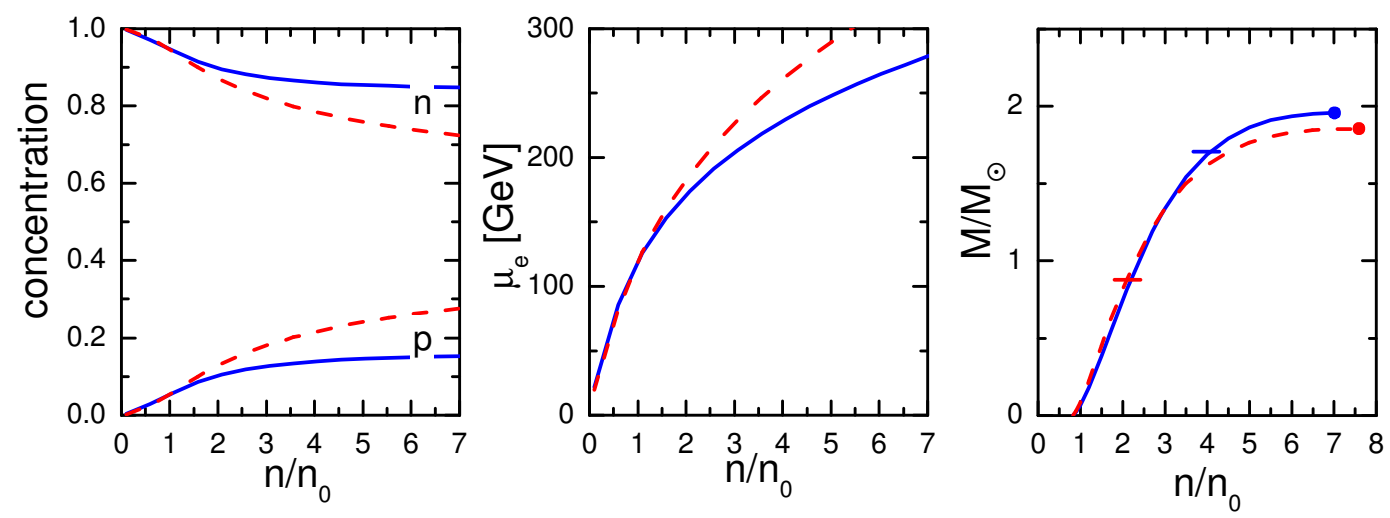

Fig. 9. The proton and neutron concentrations (left panel), and the electron chemical potential (middle panel) in the neutron star matter for the very same models as in Fig. 8. The right panel shows the mass of the neutron star as a function of the central density. The DU thresholds are indicated by cuts.

is essentially higher than the averaged value of the NS mass measured in the NS binaries. Thus we removed the problem with the low critical density for the DU process.

\subsubsection{Fit to $A 18+\delta v+U I X^{*}$ and $H H J$ EoS}

Now let us show the efficiency of the MW(nu) models on another example. In ref. [16] a parameterization of the nuclear EoS was proposed (we called it the HHJ EoS), which is a good fit to the realistic EoS of the Urbana-Argonne group [2] (A18+ $\left.\delta v+\mathrm{UIX}^{*}\right)$ up to $4 n_{0}$. For larger densities the Urbana-Argonne EoS based on non-relativistic interaction produces a supersonic velocity. The HHJ EoS treats this problem, smoothly incorporating the causality constraint. In this parameterization the energy density is given by

$$
\begin{aligned}
& E\left[n_{p}, n_{n}\right]=\left(n_{p}+n_{n}\right)\left[e_{\mathrm{B}} u \frac{2.2-u}{1+0.2 u}+a_{\mathrm{sym}} u^{0.6} \frac{\left(n_{p}-n_{n}\right)^{2}}{\left(n_{p}+n_{n}\right)^{2}}\right], \\
& u=\left(n_{p}+n_{n}\right) / n_{0}, \quad e_{B}=-15.8 \mathrm{MeV} \quad a_{\mathrm{sym}}=32 \mathrm{MeV} .
\end{aligned}
$$

In ref. [11] it was demonstrated that the HHJ EoS can be successfully fitted by the MW model, which uses as an input set

$$
\begin{aligned}
& e_{\mathrm{B}}=-15.8 \mathrm{MeV}, K=250 \mathrm{MeV}, m_{N}^{*}\left(n_{0}\right)=0.8 m_{N}, \\
& a_{\text {sym }}=28 \mathrm{MeV} .
\end{aligned}
$$

The corresponding parameter set 

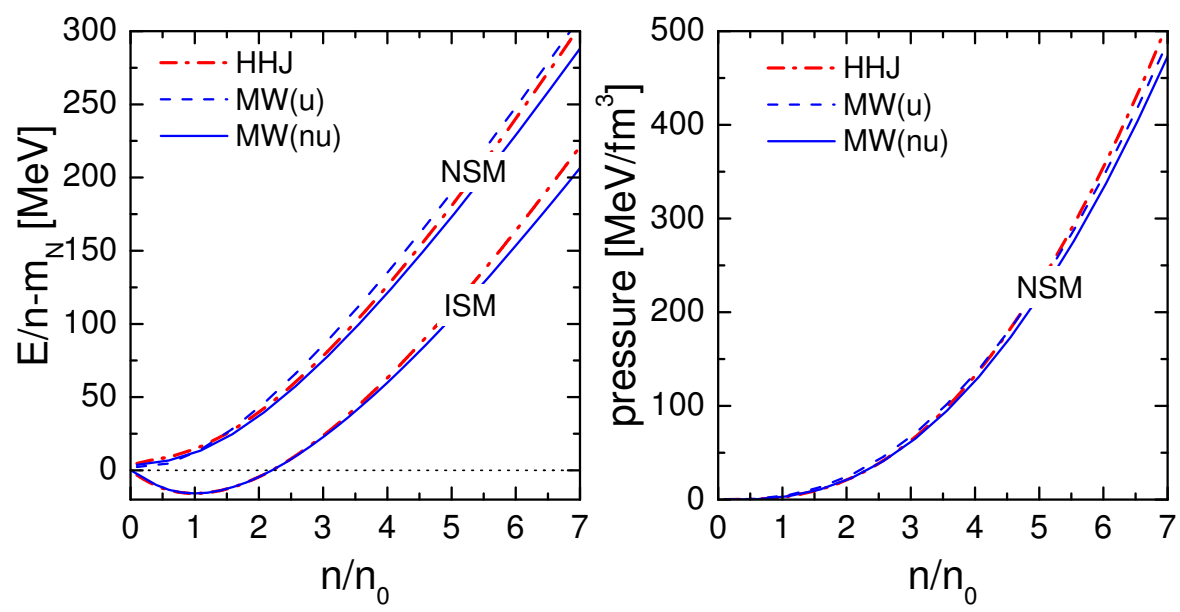

Fig. 10. Left panel: the energy per particle of the isospin symmetrical matter (ISM) and the neutron star matter (NSM), as a function of the nucleon density. Right panel: the pressure of the neutron star matter. Solid lines show calculations done within the MW(nu) model with the scaling (63) and $z=2.9$ with the input-parameter set $(61,62)$. Dashed lines correspond to the MW $(u)$ model $(z=0)$ for the very same input-parameter set. Dash-dotted lines are for the HHJ EoS (60).

$$
\begin{aligned}
& C_{\sigma}^{2}=195.10, \quad C_{\omega}^{2}=90.911, \quad C_{\rho}^{2}=77.276, \\
& b=8.6964 \times 10^{-3}, \quad c=8.1411 \times 10^{-3} .
\end{aligned}
$$

is slightly different from the original one used in ref. [11], where the nucleon mass was taken to be $938.918 \mathrm{MeV}$ instead of $938 \mathrm{MeV}$, that we adopted here. The MW(u) model with such parameters gives approximately the same dependence of the NS mass on the central density as the HHJ EoS [10]. However, as other RMF models, this MW(u) model yields a low threshold density for DU processes, $n_{\text {crit }}^{\mathrm{DU}} \simeq 2.6 n_{0}$ (corresponding to the NS mass $M \simeq 1.1 M_{\odot}$ ), whereas for the HHJ EoS $n_{\text {crit }}^{\mathrm{DU}} \simeq 5.2 n_{0}\left(M \simeq 1.8 M_{\odot}\right)$. This shortage can be corrected if we allow for a non-universal scaling of the rho meson mass and the coupling constant, which results in

$$
\eta_{\sigma}=\eta_{\omega}=1, \quad \eta_{\rho}(f)=\left(\frac{1+z f}{1+z f_{0}}\right)^{2} .
$$

For $z=2.9$ the scaling ratio $\Phi_{\rho} / \chi_{\rho}$ changes from unity at $n_{0}$ to $\simeq 1.4$ at $7 n_{0}$. Thus a dis-balance of the universal scaling that we have introduced is not too strong. The threshold density for the DU processes is now shifted to $n_{\mathrm{crit}}^{\mathrm{DU}}=5.2 n_{0}$, in accordance with that for the HHJ EoS.

Fig. 10 (left panel) demonstrates the energy per particle of the isospin symmetrical matter and of the neutron star matter (NSM) calculated within the $\mathrm{MW}(\mathrm{nu})$ model with the scaling (63) for $z=2.9$ and for the input-parameter set $(61,62)$ in comparison with the values calculated for the MW(u) model $(z=0)$, with the same input-parameter set, $(61,62)$, and for the HHJ EoS. 

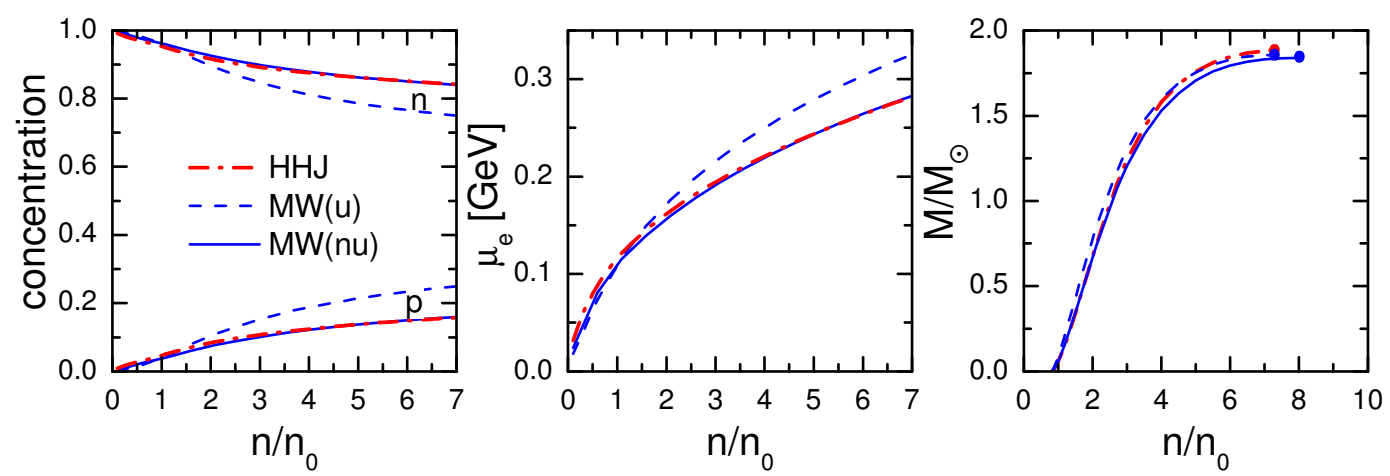

Fig. 11. The proton and neutron concentrations (left panel), the electron chemical potential in the neutron star matter (middle panel) and the mass of the neutron star as a function of the central density for the very same models as in Fig. 10.

Dashed and solid curves are not distinguishable. The right panel shows the pressure, as a function of the density for the NSM. We see that in both cases (ISM and NSM) the thermodynamic characteristics of the given MW(nu) and $\mathrm{MW}(\mathrm{u})$ models are very closed to the values calculated for the HHJ EoS.

In Fig. 11 (left and middle) we illustrate how the scaling (63) allows to change the composition of the NS matter. We see that for the given MW(nu) model (input-parameter set $(61,62))$ the proton concentration and the electron chemical potential are strongly reduced compared to the corresponding $\mathrm{MW}(\mathrm{u})$ model (the same input-parameter set) and become equal to those for the HHJ parameterization. Right panel shows NS masses as functions of the central densities for the given three models. They are very close to each other.

Concluding this section, we have shown an example of the MW(nu) model that allows to increase the limiting NS mass and to shift the DU threshold to higher densities. We have also constructed another MW(nu) model that can be used as a RMF model equivalent to the HHJ parameterization, thus also demonstrating an appropriate fit to the $A 18+\delta v+U I X^{*}$ EoS.

\section{Second order phase transition to $\rho^{-}$condensate in NS}

Now we turn to the discussion of the isospin asymmetrical matter where, as we will show, mean fields of charged $\rho$ mesons may have finite values. As we have mentioned, usually only one component, $\rho_{0}^{(3)}$ is assumed to be non-zero. A new ansatz for the $\rho$ meson fields was proposed in [26]. Besides the traditional $\rho_{0}^{(3)} \neq 0$ field it includes also spatial components of charged $\rho$ meson fields, $\rho_{i}^{ \pm}=\left(\rho_{i}^{(1)} \pm i \rho_{i}^{(2)}\right) / \sqrt{2} \neq 0, i=1,2,3$. As it was argued in ref. [26], the presence of the fields $\rho_{i}^{(3)}, \rho_{0}^{(i)}$ results in an increase of the system energy. Therefore, these fields can be put equal to zero. It was also found that the 
condition $\left(\rho_{i}^{+} \rho_{j}^{-}-\rho_{i}^{-} \rho_{j}^{+}\right)=0$ minimizes the energy. This implies that the ratio $\rho_{i}^{+} / \rho_{i}^{-}$is constant, independent of the spatial index $i$. Then we may take $\rho_{i}^{-}=a_{i} \rho_{c}$ and $\rho_{i}^{+}=a_{i} \rho_{c}^{\dagger}$, where $\vec{a}=\left\{a_{i}\right\}$ is the spatial unit vector, and $\rho_{c}$ is a complex amplitude of a charged $\rho$-meson field.

In terms of the $\rho_{0}^{(3)}$ and $\rho_{c}$ fields the corresponding contribution to the density of the thermodynamic potential (effective energy) (21) becomes

$$
\begin{aligned}
\Omega_{\rho}\left[n_{n}, n_{p} ; f, \rho_{0}^{(3)}, \rho_{c}\right] & =\frac{1}{2} g_{\rho} \chi_{\rho}\left(n_{p}-n_{n}\right) \rho_{0}^{(3)}-\frac{1}{2}\left(\rho_{0}^{(3)}\right)^{2} m_{\rho}^{2} \Phi_{\rho}^{2} \\
& -\left[\left(g_{\rho} \chi_{\rho}^{\prime} \rho_{0}^{(3)}-\mu_{c h}^{\rho}\right)^{2}-m_{\rho}^{2} \Phi_{\rho}^{2}\right]\left|\rho_{c}\right|^{2} .
\end{aligned}
$$

Variation of the Lagrangian/(thermodynamic potential) with respect to the fields $\rho_{0}^{(3)}$ and $\rho_{i}^{-}$yields equations of motion

$$
\begin{aligned}
& {\left[\left(g_{\rho} \chi_{\rho}^{\prime} \rho_{0}^{(3)}-\mu_{c h}^{\rho}\right)^{2}-m_{\rho}^{2} \Phi_{\rho}^{2}\right] \rho_{c}=0} \\
& m_{\rho}^{2} \Phi_{\rho}^{2} \rho_{0}^{(3)}+2 g_{\rho} \chi_{\rho}^{\prime}\left(g_{\rho} \chi_{\rho}^{\prime} \rho_{0}^{(3)}-\mu_{c h}^{\rho}\right)\left|\rho_{c}\right|^{2}=\frac{1}{2} g_{\rho} \chi_{\rho}\left(n_{p}-n_{n}\right) .
\end{aligned}
$$

This system of equations has two solutions. The first solution is the traditional one, cf. (26), (27),

$$
\rho_{0}^{(3)}=\frac{1}{2} \frac{g_{\rho}}{m_{\rho}^{2}} \frac{\chi_{\rho}}{\Phi_{\rho}^{2}}\left(n_{p}-n_{n}\right), \quad \rho_{c}=0,
$$

yielding the contribution to the density of the thermodynamic potential

$$
\Omega_{\rho}^{(1)}\left[n_{n}, n_{p} ; f\right]=\frac{C_{\rho}^{2}\left(n_{n}-n_{p}\right)^{2}}{8 m_{N}^{2} \eta_{\rho}(f)} .
$$

The second solution of (65) (found previously in [26], neglecting $\mu_{c h}^{\rho}$, and without scaling of couplings) is as follows

$$
\begin{aligned}
\rho_{0}^{(3)} & =\frac{\mu_{c h}^{\rho}}{g_{\rho} \chi_{\rho}^{\prime}}-\frac{m_{\rho}}{g_{\rho}} \frac{\Phi_{\rho}}{\chi_{\rho}^{\prime}} \operatorname{sign}\left(n_{n}-n_{p}\right), \quad\left|\rho_{c}\right|^{2}=\frac{\left|n_{p}-n_{n}\right|-n^{\rho}}{4 m_{\rho} \eta_{\rho}^{1 / 2} \chi_{\rho}^{\prime}} \\
n^{\rho} & =2 \frac{m_{\rho} m_{N}^{2}}{C_{\rho}^{2}} \frac{\eta_{\rho}^{1 / 2} \Phi_{\rho}^{2}}{\chi_{\rho}^{\prime}}\left(1-\frac{\mu_{c h}^{\rho}}{m_{\rho} \Phi_{\rho}} \operatorname{sign}\left(n_{n}-n_{p}\right)\right) .
\end{aligned}
$$

It exists only, if $\left|n_{n}-n_{p}\right|>n^{\rho}$. The density of the thermodynamic potential corresponding to this solution takes the form 


$$
\Omega_{\rho}^{(2)}\left[n_{n}, n_{p} ; f\right]=\Omega_{\rho}^{(1)}\left[n_{n}, n_{p} ; f\right]-\frac{C_{\rho}^{2}}{8 m_{N}^{2} \eta_{\rho}}\left(\left|n_{n}-n_{p}\right|-n^{\rho}\right)^{2} .
$$

Hence, for $n>n_{\mathrm{c}}^{\rho, \text { II }}$, where the value $n_{\mathrm{c}}^{\rho \text {,II }}$ follows from the solution of the equation

$$
n^{\rho}=\left|n_{n}-n_{p}\right|
$$

the second solution (69) becomes energetically favorable compared to the traditional solution (66). The density $n_{\mathrm{c}}^{\rho \text {,II }}$ which can be written as

$$
n_{\mathrm{c}}^{\rho, \mathrm{II}}=2 \frac{m_{\rho} m_{N}^{2}}{C_{\rho}^{2}} \frac{\eta_{\rho}^{1 / 2} \Phi_{\rho}^{2}}{\left(1-2 Y_{p}^{(c)}\right) \chi_{\rho}^{\prime}}\left(1-\frac{\mu_{c h}^{\rho}}{m_{\rho} \Phi_{\rho}}\right), \quad Y_{p}^{(c)}=\frac{n_{p}}{n_{\mathrm{c}}^{\rho, \mathrm{II}}}<\frac{1}{2},
$$

is the critical density with respect to a second-order phase transition. In principle a transition to a new solution could be of a first order with an abrupt change of the isospin composition and the density. It would then happen at a smaller critical density $n=n_{\mathrm{c}}^{\rho, \mathrm{I}}<n_{\mathrm{c}}^{\rho, \mathrm{II}}$, cf. [26]. We studied this possibility (see below) and found out that in the presence of electrons the first-order phase transition is not realized. Thus we conclude that in the frameworks of the RMF models that we exploit here and with taking into account of electrons the charged $\rho$-meson condensation occurs by the second-order phase transition. Note that the new solution (69) induces a spontaneous breaking of the spatial symmetry of the system, since we fixed only one value $\rho_{c}$, whereas the choice of components $a_{i}$ is not fixed.

The charged $\rho$-meson chemical potential is found from the chemical equilibrium conditions. Considering the reaction $e+n \leftrightarrow \rho^{-}+n$ we find

$$
\mu_{c h}^{\rho}=\mu_{e}
$$

The number of protons, electrons, muons and $\rho^{-}$is governed by the local charge neutrality condition

$$
n_{p}-n_{e}-n_{\mu}+n_{c h}^{\rho}=0, \quad n_{c h}^{\rho}=2\left(g_{\rho} \chi_{\rho}^{\prime} \rho_{0}^{(3)}-\mu_{c h}^{\rho}\right)\left|\rho_{c}\right|^{2}<0 .
$$

In the phase with $\rho_{c} \neq 0$ from $(17)$, (69) we find

$$
\begin{aligned}
& \mu_{e}=\frac{1}{1+\chi_{\rho}(\bar{f}) / \chi_{\rho}^{\prime}(\bar{f})} \\
& \times\left(\sqrt{m_{N}^{2} \Phi_{N}^{2}(\bar{f})+p_{\mathrm{F}, n}^{2}}-\sqrt{m_{N}^{2} \Phi_{N}^{2}(\bar{f})+p_{\mathrm{F}, p}^{2}}+m_{\rho} \frac{\Phi_{\rho}(\bar{f}) \chi_{\rho}(\bar{f})}{\chi_{\rho}^{\prime}(\bar{f})}\right),
\end{aligned}
$$




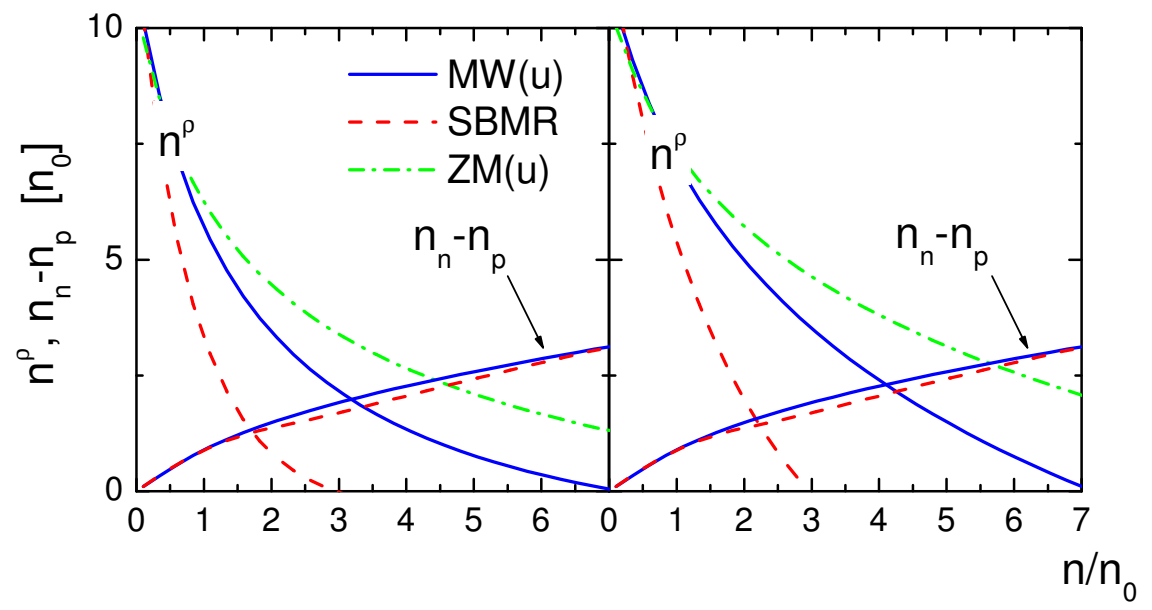

Fig. 12. Densities $n^{\rho}$ from (69) and $n_{n}-n_{p}$ calculated for the neutron star matter within the MW(u) (input-parameter set $(29,37,38)$ ), ZM(u) (input-parameter set $(29,40))$ and SBMR models. Left panel shows the results for the choice $\chi_{\rho}^{\prime}=1$ and right panel, for $\chi_{\rho}^{\prime}=\Phi_{\rho}$. Crossing points of curves $n^{\rho}$ with the curve $n_{n}-n_{p}$ correspond to $n=n_{\mathrm{c}}^{\rho, \mathrm{II}}$, cf. eq. (70).

$$
\begin{aligned}
& n_{p}=\frac{\left(\mu_{e}^{2}-m_{e}^{2}\right)^{\frac{3}{2}}}{3 \pi^{2}} \theta\left(\mu_{e}-m_{e}\right)+\frac{\left(\mu_{e}^{2}-m_{\mu}^{2}\right)^{\frac{3}{2}}}{3 \pi^{2}} \theta\left(\mu_{e}-m_{\mu}\right)+n_{c h}^{\rho}\left(\mu_{e}, n_{p}, n_{n}, \bar{f}\right), \\
& n_{c h}^{\rho}=2 m_{\rho} \Phi_{\rho}(f)\left|\rho_{c}\right|^{2} .
\end{aligned}
$$

The main uncertainty of our consideration is the unknown modification of the non-Abelian $\rho$-meson self-interaction in nuclear medium, which in our scheme is encoded in the scaling parameter $\chi_{\rho}^{\prime}$. As we have mentioned, we see no reason for the scaling of $\chi_{\rho}^{\prime}$, thus taking $\chi_{\rho}^{\prime}=1$. However for the completeness of the consideration and in order to investigate the sensitivity of our results to the parameter variation we consider also the case $\chi_{\rho}^{\prime} \simeq \chi_{\rho} \simeq \Phi_{\rho}$. Moreover note that some QCD motivated studies, cf. [27], use smaller values for the $\rho$ meson coupling constants $g_{\rho}=g_{\rho}^{\prime} \simeq m_{\rho} / F_{\pi} \simeq 5.8, F_{\pi} \simeq 132 \mathrm{MeV}$, than those follow from the fits in the RMF models. In this respect the second choice $\chi_{\rho}^{\prime} \simeq \chi_{\rho} \simeq \Phi_{\rho}$ effectively demonstrates what could be if $g_{\rho}$ were decreased.

The quantity $n^{\rho}(n)$ given by (69) and $n_{n}-n_{p}$ are depicted in Fig. 12 for three types of models MW(u) (input-parameter set $(29,37,38)$ ), ZM(u) (inputparameter set $(29,40)$ ), and SBMR discussed above. We use here $m_{\rho}=770 \mathrm{MeV}$. The actual value of the critical density, $n_{\mathrm{c}}^{\rho, \mathrm{II}}$, for the second order phase transition to the charged $\rho$-meson condensate state is given by the solution (71) of eq. (70). We see that the condensation is possible at $n_{\mathrm{c}}^{\rho, \mathrm{II}}=3.2 n_{0}$ for the MW(u) model (would be $4.1 n_{0}$ for the model with the scaling of $\chi_{\rho}^{\prime}$ ), at $n_{\mathrm{c}}^{\rho \text {,II }}=4.5 n_{0}$ for the $\mathrm{ZM}(\mathrm{u})$ model $\left(5.6 n_{0}\right.$ for the model with the scaling of $\left.\chi_{\rho}^{\prime}\right)$, and at $n_{\mathrm{c}}^{\rho \text {,II }}=1.7 n_{0}$ for the SBMR model $\left(2.2 n_{0}\right.$ for the model with the scaling of $\left.\chi_{\rho}^{\prime}\right)$. 


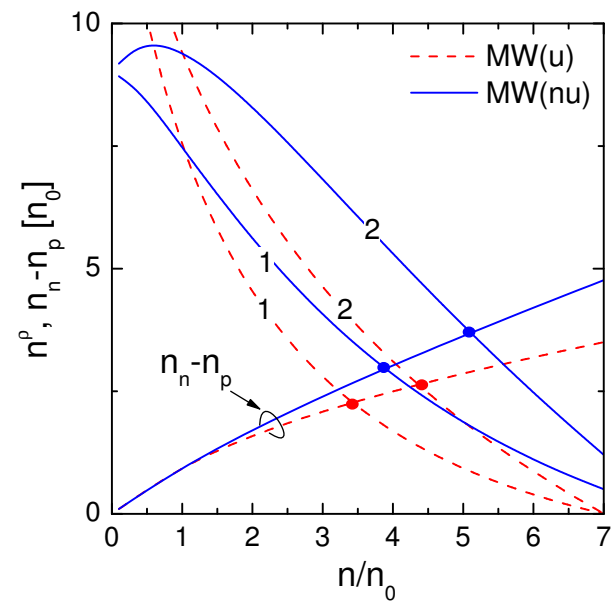

Fig. 13. Densities $n^{\rho}$ from (69) and $n_{n}-n_{p}$ calculated for the neutron star matter within the $\mathrm{MW}(\mathrm{nu})$ model (63), $z=2.9$ (the input-parameter set (61); (62)) are shown by solid lines. Dashed lines are for $z=0$ and the same choice of other parameters. Fat dots mark the critical densities $n_{\mathrm{c}}^{\rho \text {,II }}$. Curves 1 correspond to $\chi_{\rho}^{\prime}=1$, whereas 2 , to $\chi_{\rho}^{\prime}=\Phi_{\rho}$.

In Fig.13 we show the same, as in Fig. 12, but for the MW(nu) model with $z=2.9$ specified by $(61,62,63)$. As the consequence of $\eta_{\rho}>1$, the MW(nu) model produces a higher critical density for the charged $\rho$ meson condensation, $n_{c}^{\rho, \mathrm{II}} \simeq 3.9 n_{0}$ for $\chi_{\rho}^{\prime}=1$ and $n_{c}^{\rho, \mathrm{II}} \simeq 5.1 n_{0}$ for $\chi_{\rho}^{\prime}=\Phi_{\rho}$. This is basically due to the smaller value of the asymmetry energy in (61) and therefore, the smaller value of the coupling constant $C_{\rho}$. We point out that in a most realistic cases of the $\mathrm{MW}(\mathrm{u})$ and $\mathrm{MW}(\mathrm{nu})$ models the novel charged $\rho$ meson condensation appears already for sufficiently low densities $n \sim(3 \div 4) n_{0}$. As the price for the increase of the threshold density for the DU process, the critical density for the condensation increased in case of this MW(nu) model. The MW(nu) model (56) for $z=0.65$, using the input-parameter set $(29,57,58)$ yields $n_{c}^{\rho \text { II }} \simeq 3.4 n_{0}$ for $\chi_{\rho}^{\prime}=1$ and $n_{c}^{\rho, \mathrm{II}} \simeq 4.6$ for $\chi_{\rho}^{\prime}=\Phi_{\rho}$.

The critical density, $n_{\mathrm{c}}^{\rho \text {,II }}$ depends on the particular values of the effective nucleon mass and compressibility at saturation, which are used to constrain parameters of the RMF model. We study these dependences on the example of the MW(u) model, which allows for independent variation of the input parameters $K$ and $m_{N}^{*}\left(n_{0}\right) / m_{N}$. We calculate the critical density $n_{\mathrm{c}}^{\rho \text {,II }}$, as a function of the input parameter $m_{N}^{*}\left(n_{0}\right) / m_{N}$ for $K=200 \mathrm{MeV}$ and $300 \mathrm{MeV}$ for two choices of the $\chi_{\rho}^{\prime}$ scaling mentioned above. Results are presented in Fig. 14. We see that $n_{\mathrm{c}}^{\rho \text {,II }}$ is weakly dependent on $K$ but varies strongly with the effective nucleon mass, especially for $m_{N}^{*}\left(n_{0}\right) / m_{N} \gtrsim 0.75$. For $0.6 \lesssim m_{N}^{*}\left(n_{0}\right) / m_{N} \lesssim 0.8$ the critical density varies within the interval $n_{0} \lesssim n_{\text {c II }} \lesssim 3 n_{0}$ for $\chi_{\rho}^{\prime}=1$.

Fig. 15 demonstrates the energy per particle and the pressure of the NS matter as a function of the nucleon density without (dash curves) and with $\rho^{-}$condensate (solid curves) for the case of the MW(u) model (the input-parameter 

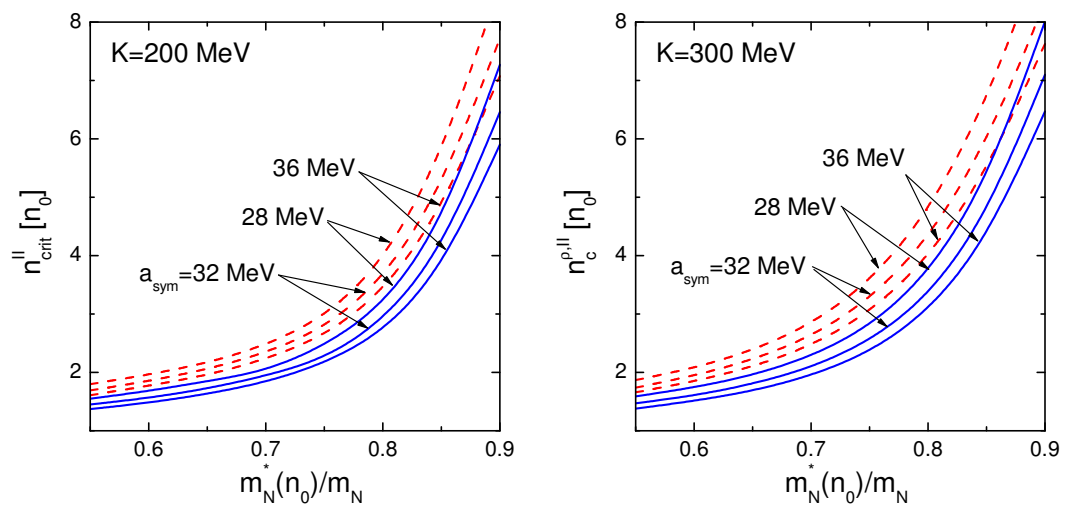

Fig. 14. The critical density of the charged $\rho$ meson condensation, $n_{\mathrm{c}}^{\rho \text {, II }}$ as a function of the $m_{N}^{*}\left(n_{0}\right)$ used as an input of the MW(u) model for two values of the compressibility modulus $K$ and three values of $a_{\text {sym }}$. Other input values are taken according to (29). The solid lines are calculated for the choice $\chi_{\rho}^{\prime}=1$ and the dashed ones for $\chi_{\rho}^{\prime}=\Phi_{\rho}$.
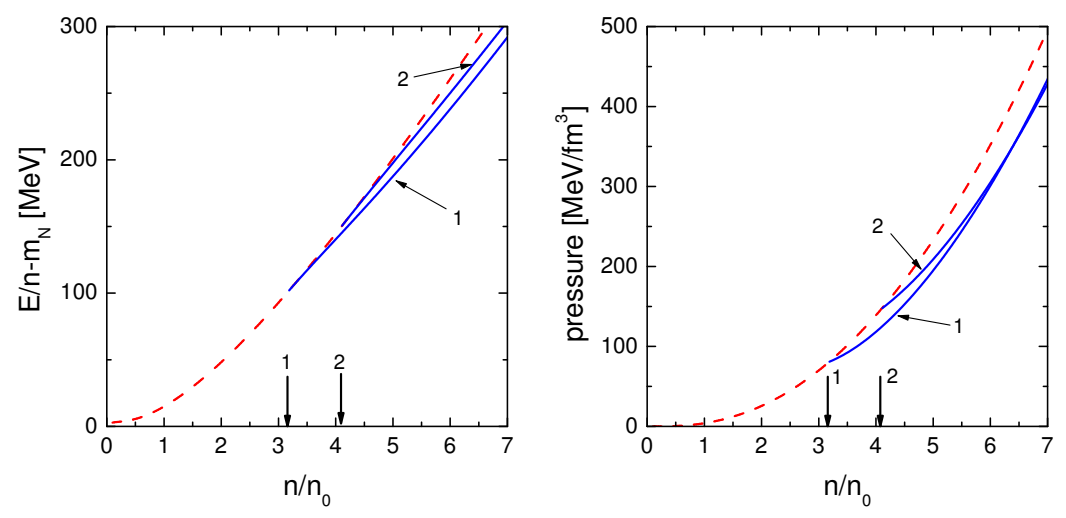

Fig. 15. The energy per particle (left panel) and the pressure (right panel) of the neutron star matter as a function of the nucleon density without (dash curves) and with (solid curves) $\rho^{-}$condensate for the MW(u) model (the input-parameter set (29), (37); (38)). The lines labeled by "1" are calculated for the choice $\chi_{\rho}^{\prime}=1$ and the lines labeled by "2" are for $\chi_{\rho}^{\prime}=\Phi_{\rho}$.

set $(29,37,38))$ cf. Figs. 2, 3. Curve 1 is for $\chi_{\rho}^{\prime}=1$ and curve 2, for $\chi_{\rho}^{\prime}=\Phi_{\rho}$. We observe no van der Waals behavior of the pressure typical for the first order phase transitions. The EoS is softened in the presence of the second order phase transition to the charged $\rho^{-}$condensate but quantitatively the softening effect is minor.

In Fig. 16 we show the proton fraction $Y_{p}=n_{p} / n$, the electron chemical potential, and the NS mass for the NS matter as a function of the nucleon density without (dash curves) and with (solid curves) $\rho^{-}$condensate for the case of the MW(u) model (the input-parameter set $(29,(37,38))$, cf. Fig. 4. Curves 1 are for $\chi_{\rho}^{\prime}=1$ and curves 2 are for $\chi_{\rho}^{\prime}=\Phi_{\rho}$. We see that a part of electrons is now replaced to the condensate $\rho^{-}$mesons. Due to that the electron chemical potential decreases in presence of the condensate compared 

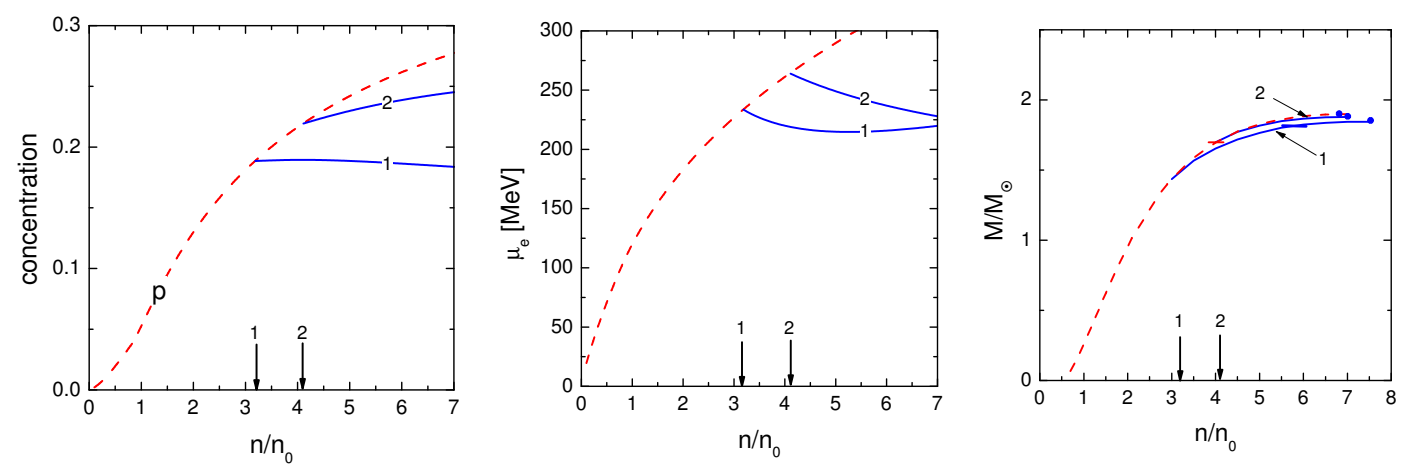

Fig. 16. The proton concentration (left panel) and the electron chemical potential (middle panel) of the neutron star matter as a function of the nucleon density without (dash curves) and with (solid curves) $\rho^{-}$condensate for the MW(u) model (the input-parameter set $(29,37,38)$ ). The right panel shows the mass of the neutron star as a function of the central density. The lines labeled by "1" are calculated for the choice $\chi_{\rho}^{\prime}=1$ and the lines labeled by "2" are for $\chi_{\rho}^{\prime}=\Phi_{\rho}$.
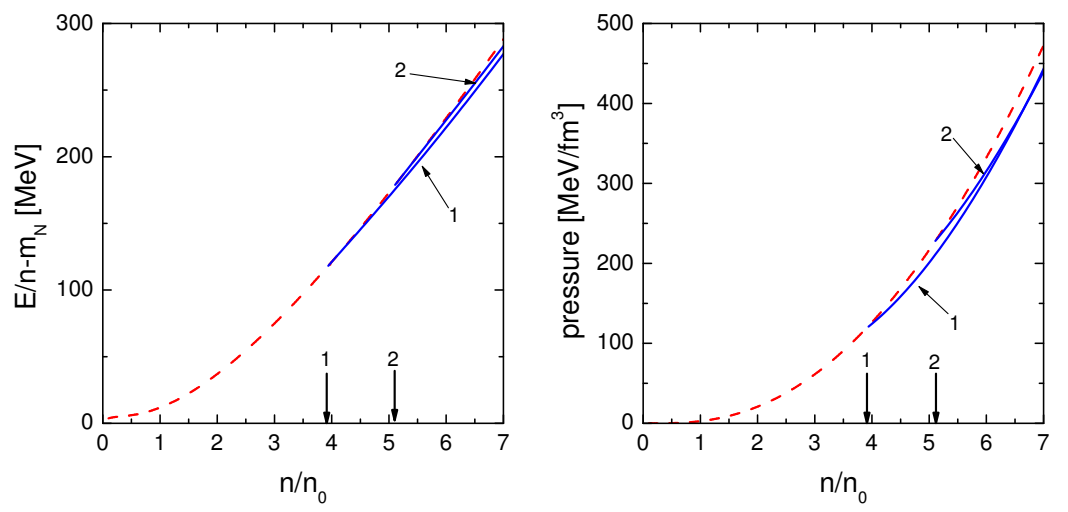

Fig. 17. The same as in Fig. 15 but for the MW(nu) model with (63), $z=2.9$, the input-parameter set $(61,62)$.

to the case without the condensate. The proton fraction becomes smaller in the presence of the $\rho^{-}$condensate than would be in the absence of the condensate. However, as we obtain in the framework of the $M W(u)$ model, DU processes once started at $2.07 n_{0}$ stay operative in the condensate phase up to rather high densities (up to $5.8 n_{0}$ for case 1 and up to the maximum central density for case 2 ). The neutron star masses change very little with the appearance of the condensate.

Figure 17 shows the same as Figure 15 but for the MW(nu) model with (63), $z=2.9$, the input-parameter set $(61,62)$. Again we find no van der Waals behavior of the pressure and only weak softening of the EoS. The proton concentration, electron chemical potential and the NS masses for this model are shown in Fig. 18. The only qualitative difference is seen in the behavior of the proton fraction for the case $\chi_{\rho}^{\prime}=\Phi_{\rho}$. It increases with increase of the density in this particular case. However the triangle inequality for the DU processes is not fulfilled since a part of the negative charge is taken by the $\rho^{-}$. 

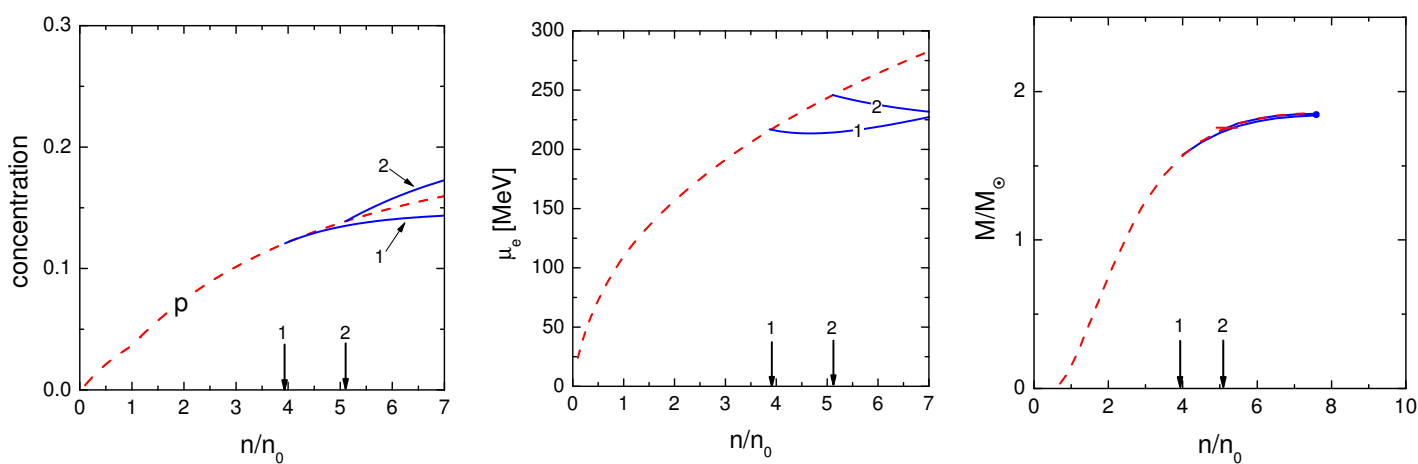

Fig. 18. The same as in Fig. 16 but for the MW(nu) model with (63), $z=2.9$, the input-parameter set $(61,62)$.
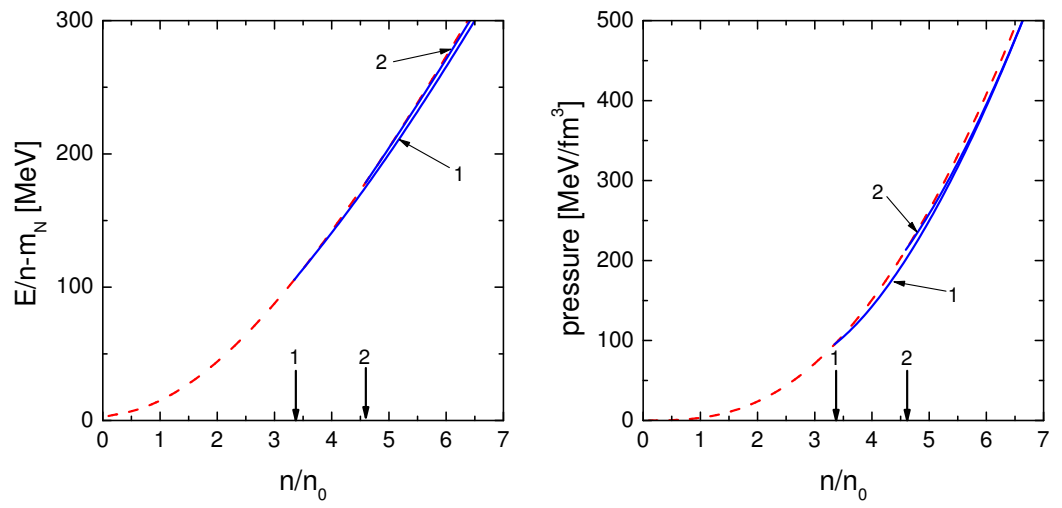

Fig. 19. The same as in Fig. 15 but for the MW(nu) model (56), $z=0.65$, the input-parameter set $(29,57,58)$.
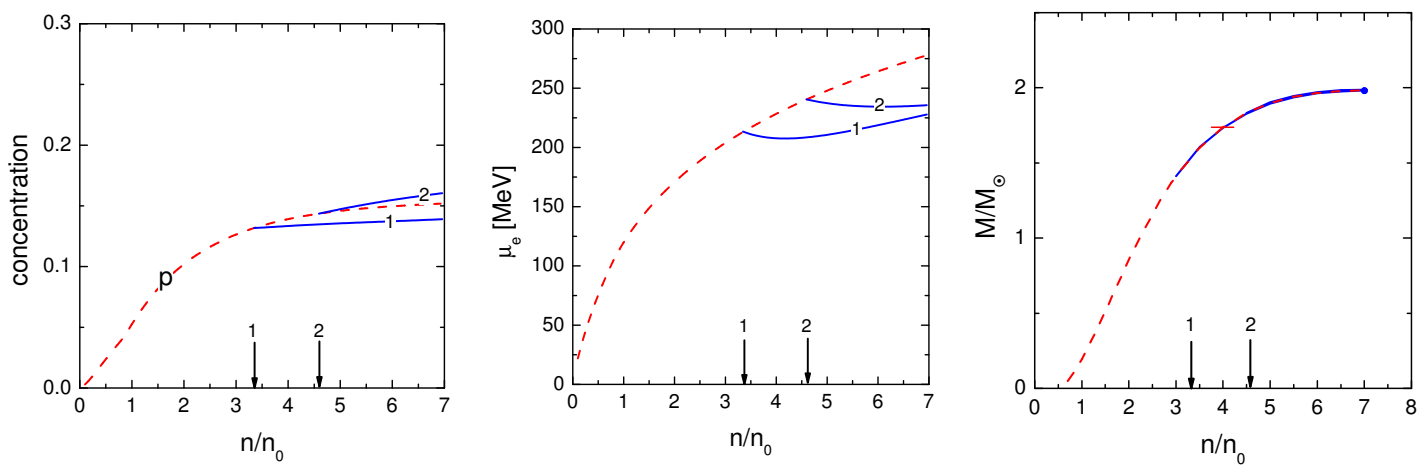

Fig. 20. The same as in Fig. 16 but for the MW(nu) model (56), $z=0.65$, the input-parameter set $(29,57,58)$.

Thus in the case of the $M W(n u)$ model, $z=2.9$, DU processes do not occur in the phase with the condensate at all.

Fig. 19 shows the same as Fig. 17 but for the MW(nu) model (56), $z=0.65$, the input-parameter set $(29,57,58)$. Fig. 20 shows the same as Fig. 18 for the given model. Qualitative behavior of the quantities for the given $\mathrm{MW}(\mathrm{nu})$ model (56) with $z=0.65$ is the same as for the MW(nu) model (63) with 
$z=2.9$. Also in the case of the $M W(n u)$ model, $z=0.65, D U$ processes are non-operative in the condensate phase for both cases $\chi_{\rho}^{\prime}=1$ and $\chi_{\rho}^{\prime}=\Phi_{\rho}$.

Concluding this section we found that a new phase of the dense nuclear matter with a charged $\rho$ meson mean field might exist in the NS interiors already for not too high densities. For the most realistic cases we found $n_{\mathrm{c}}^{\rho \text {,II }} \lesssim 4 n_{0}$.

\section{Neutrino emissivity in the DU processes on $\rho^{-}$condensate}

As we have demonstrated, the standard DU processes $n \rightarrow p e \bar{\nu}$, pe $\rightarrow n \nu$ are forbidden in cases of the MW(nu) models $(56,58)$ and $(63,62)$ up to sufficiently high density $\left(n>4 n_{0}\right.$ and $n>5.2 n_{0}$ for the case $\left.\chi_{\rho}^{\prime}=1\right)$. Therefore we consider these models as most realistic ones. However a new channel of the DUlike processes on the condensate charged $\rho^{-}$meson, e.g., $n \rho_{c}^{-} \rightarrow n e \bar{\nu}$ becomes operative for $n>n_{c}^{\rho \text { II }}$. Since the charged $\rho^{-}$condensate arises in the s-wave, the calculation of its contribution to the emissivity can be done similar to that for the kaon condensation processes, except additional requirement of the chiral symmetry used in ref. [46]. Note that ref. [46] did not take into account nucleon-nucleon correlations in vertices that yields an additional suppression factor $\lesssim 10^{-1}$ for all condensate processes, cf. [47,5,48]. Recovering the missing factor we easily estimate the neutrino emissivity

$$
\epsilon_{\nu}^{\rho_{c}^{-}} \sim 10^{26} g_{\rho N}^{2} \chi_{\rho}^{2}\left(\frac{m_{N}^{*}}{m_{N}}\right)^{2}\left(\frac{\mu_{e}}{m_{N}}\right) T_{9}^{6} \frac{n_{c h}^{\rho}}{m_{\rho} \Phi_{\rho} m_{\pi}^{2}} \Gamma^{4}, \quad \frac{\mathrm{erg}}{\mathrm{cm}^{3} \cdot \mathrm{sec}},
$$

$m_{\pi}=140 \mathrm{MeV}, T_{9}=T / 10^{9} \mathrm{~K}, \Gamma^{4}=\Gamma_{s}^{2} \Gamma_{w-s}^{2}$ is the nucleon-nucleon correlation factor,

$$
\begin{aligned}
& \Gamma_{s}=\widetilde{\Gamma}\left(f^{\prime}, \varepsilon \simeq \mu_{e}, k \simeq p_{\mathrm{Fn}}\right), \quad \widetilde{\Gamma}(x, \varepsilon, k)=\left[1-4 C_{0} x A_{n p}(\varepsilon, k)\right]^{-1}, \\
& \Gamma_{w-s}^{2}=\widetilde{\Gamma}^{2}\left(f^{\prime}, \varepsilon=q \simeq p_{\mathrm{Fe}}\right),
\end{aligned}
$$

$C_{0} \simeq 0.77 m_{\pi}^{-2}$ relates to the density of states at the Fermi surface for $n=n_{0}$, $f^{\prime} \simeq 0.5 \div 1$ is the Landau-Migdal parameter in isospin channel, cf. [49,5,50], $A_{n p}$ is the proton - neutron hole loop (without spin degeneracy factor), see [48]. As we have mentioned, the presence of the correlation factor is crucially important. It may suppress the rate by a factor $\sim 10^{-1} \div 10^{-2}$. Another suppression factor arises from a small concentration $n_{c h}^{\rho}$ in the new phase, $n_{c h}^{\rho}<n_{p}$.

In presence of the nucleon superfluidity the emissivity of the DU-like processes is additionally suppressed by a factor $\min \left[\xi_{n n}, \xi_{p p}\right], \xi_{i i} \sim e^{-\Delta_{i i} / T}$ for $T<T_{c i}$, where $\Delta_{i i}$ is the pairing gap in the corresponding nn or pp channel. According 
to $[51,10]$ the ${ }^{3} P_{2} n n$-pairing gap should be very small, less than $10 \mathrm{keV}$. If so, $\min \left[\xi_{n n}, \xi_{p p}\right] \simeq \xi_{p p}$. Final rough estimation of the emissivity is

$$
\epsilon_{\nu}^{\rho_{c}^{-}} \sim\left(10^{24} \div 10^{25}\right) T_{9}^{6} \min \left[\xi_{n n}, \xi_{p p}\right] \frac{\mathrm{erg}}{\mathrm{cm}^{3} \cdot \mathrm{sec}}
$$

This rate is typically either smaller or of the order of that expected for the charged pion condensate. However it is much smaller than that for the standard DU processes, if the latter were permitted. On the other hand, for typical densities and temperatures $T \sim 0.3 \div 3 \cdot 10^{8} \mathrm{~K}$ the emissivity on the charged $\rho^{-}$ condensate is somewhat larger than the emissivity of the competing mediummodified Urca processes, as they have been computed in $[52,10]$.

As has been shown in ref. [10] within HHJ EoS, the critical density of the charged pion condensate should not be less than $(2.5 \div 2.7) n_{0}$. Otherwise even rather low mass NS would cool too fast in disagreement with the data. A similar statement can be done in case of the charged $\rho^{-}$condensation. Thus we see that the values $n_{c}^{\rho \text { II }} \sim(3 \div 4) n_{0}$ that we have obtained within our MW(u) and MW(nu) models do not contradict to the analysis of the NS cooling [10].

\section{Conclusion}

In this paper we studied generalized relativistic mean field models that include in-medium changes of meson masses and coupling constants. We demonstrated equivalence of a certain class of the models. Practically we showed that the energy density for homogeneous matter depends only on three independent combinations of mass and coupling scaling functions. Often this equivalence is not realized and one considers some models as different, whereas in reality they are identical for the description of homogeneous matter on the mean field level.

We showed that a naive modification of solely boson masses would lead to severe drawbacks, like discontinuous changes of mean fields and hadron masses with the density. To get rid off these problems the mass scaling should be essentially compensated by the scaling of the corresponding coupling constants.

For linear RMF models, i.e., without a non-Abelian $\rho$ interaction and a nonlinear $\sigma$ potential $U$, we demonstrated equivalence of the models with universal scaling $g_{i}^{*} m_{i} / g_{i} m_{i}^{*} \simeq 1, i=\sigma, \omega, \rho$, and the models without any scaling of those masses and couplings. For non-linear RMF models $(U \neq 0)$ the above equivalence would require a certain relation between corresponding non-linear potentials. 
We have demonstrated the efficiency of the generalized relativistic mean field models based on the modified Walecka (MW) model with a non-universal scaling of meson masses and couplings $(\mathrm{MW}(\mathrm{nu}))$. It allows to enlarge the threshold density for the direct Urca process and produces a stiffer equation of state without any changes of the EoS near the saturation density. These possibilities are favored by "the nuclear medium cooling scenario" for the neutron star cooling, cf. [10] and by recent measurements of heavy neutron stars, cf. [12].

Our generalized mean field models with a non-universal scaling of meson masses and couplings might be useful in discussion of possibilities of different phase transitions in neutron star interiors, since they allow to compensate a softening of the equation of state due to a phase transition by a stiffening due to a stronger decrease of masses compared to a decrease of couplings. Especially the model might be helpful in discussion of hybrid stars. With the standard hadron models the quark matter either begins to appear already at rather low neutron star mass, or does not appear at all, cf. [45]. In the former case the limiting mass of the star is found to be rather low (typically $\lesssim 1.8 M_{\odot}$ ). If one used a model assuming a smaller nucleon mass at the saturation, one could get a still higher value of the limiting neutron star mass.

We also demonstrated an example of the relativistic mean field model that fits well the modern Urbana-Argonne equation of state (more precisely, the fit [16] that cures the causality problem), yielding the same threshold density for the direct Urca process.

The MW(nu) models can be useful for the description of properties of finite nuclei. Here equivalence is absent due to the spatial inhomogeneity of the field profiles. In standard MW models one is forced to decrease the $\sigma$ meson mass in order to appropriately fit the proton density profile and the binding energy as a function of the atomic number, cf. [44]. In the framework of the MW(nu) models there is still a room to fit the scaling laws for effective masses and couplings.

It would be interesting to apply the model to the description of heavy-ion collisions. Early naive studies of the pion yield in heavy-ion collisions required extremely hard EoS, with the compressibility $K \sim 1000 \mathrm{MeV}$. Further it was demonstrated, cf. $[5,53,54]$ and references therein, that the problem might be naturally solved within the standard modified Walecka model (MW) when one includes contributions from soft pion modes. These modes yield very small contribution to thermodynamical characteristics of the cold nuclear matter but produce a large contribution for the hot nuclear matter. The physical reason is obvious: soft modes are easily excited with the temperature increase. Due to a decrease of $\sigma, \omega, \rho$ and nucleon masses related modes become to be softer. Thus our mean field models may give an appropriate basis to go beyond the 
mean field level considering thermal excitations.

Recent studies of the collective isospin flow [23] extracted the density dependence of the symmetry energy but did not introduce any astrophysical constraints and a possible soft mode contribution. It is therefore a challenge to fit the corresponding data using the constructed above models with inclusion of thermal contribution from soft modes.

If one introduces a rho-meson field as a non-Abelian gauge boson (cf. [27]) in a relativistic mean-field model which supports the decreasing $\rho$-meson mass, one should take care of the possibility of the charged rho meson condensation in neutron star matter. Following [26], we found that at density $n>n_{\mathrm{c}}^{\rho \text {,II }}$ the charged $\rho$-meson mean field appears. We demonstrated that in the framework of our relativistic mean field models the novel phase arises by a second order phase transition. The critical density $n_{\mathrm{c}}^{\rho, \mathrm{II}}$ is rather low in the neutron star matter (typically $\sim 3 \div 4 n_{0}$ ), if one allows for a decrease of the rho meson mass. We also included the corresponding decrease of the effective nucleonrho coupling, as well as the scaling of other meson masses and couplings. If one used a model assuming a smaller nucleon mass at the saturation, one could get a still smaller value of the critical density for the charged $\rho$ condensation. Charged $\rho$ condensation slightly softens the equation of state. In the neutron-enriched matter the charge of the $\rho$-meson condensate is negative. We evaluated the emissivity of the direct Urca-like processes on the condensate $\rho^{-}$meson. The resulting rate behaves similar to that for the $\pi^{-}$condensate. The analysis of the cooling data indicates that the value $n_{\mathrm{c}}^{\rho \text {,II }}$ should be not too small, $n_{\mathrm{c}}^{\rho, \mathrm{II}} \gtrsim 2.5 \div 2.7 n_{0}$.

Our non-Abelian $\rho$ meson field is in some sense analogous to the gluon field. Dropping of the effective $\rho$ meson mass enforces a similarity. Effective nucleon and other meson masses also decrease with increase of the density. Decrease of the corresponding effective couplings motivates a future asymptotic freedom in the quark-gluon phase. All these features might be in favor of a smooth transition from the hadron degrees of freedom to the quark-gluon degrees of freedom. Up to now there is no appropriate description of a cross-over temperature region seen from the lattice results [55]. Formulated above models might be helpful in construction of such a description.

\section{Acknowledgments}

We would like to thank A.D. Jackson for stimulating discussions. We also thank Yu. B. Ivanov for the discussion of the results. D.N.V. highly appreciates hospitality and support of GSI Darmstadt. The work has been supported in part by DFG (project 436 Rus 113/558/0-2), and by RFBR grant NNIO-0302-04008. 


\section{A Thermodynamic potential of RMF model in presence of the electric field}

So far we have disregarded the electromagnetic interactions. But only including the coordinate dependent electric field one may properly discuss structures, that may occur at first order phase transitions in charged systems. Indeed, a charged structure may exist, only if there is an inhomogeneous (not constant) electric potential. Although we did not find a first order phase transition in the framework of our RMF model EoS, this result might be still model dependent. Here we demonstrate how one can include electromagnetic interactions into the scheme. We consider the NS matter consisting of the neutrons, protons, electrons and muons. We incorporate mean meson fields including charged $\rho$ meson field, $\rho_{\mu}^{-}=a_{\mu} \rho_{c}, \rho_{\mu}^{-}=a_{\mu} \rho_{c}^{\dagger}$ with $a_{\mu}=(0, \vec{a})$ and the electric field.

The interaction with the static electric field $e A^{\mu}=(-V, \overrightarrow{0})$ can be introduced according to the minimal coupling prescription via the gauge replacement

$$
\begin{aligned}
\partial_{\mu} \Psi_{N} & \rightarrow\left(\partial_{\mu}+\frac{i}{2} e A_{\mu}\left(1+\tau_{3}\right)\right) \Psi_{N}, \quad \partial_{\mu} \Psi_{l} \rightarrow\left(\partial_{\mu}-i e A_{\mu}\right) \Psi_{l}, \\
\vec{\rho}_{\mu \nu} & \rightarrow \vec{\rho}_{\mu \nu}-e A_{\mu}\left[\vec{n}_{3} \times \vec{\rho}_{\nu}\right]+e A_{\nu}\left[\vec{n}_{3} \times \vec{\rho}_{\mu}\right]
\end{aligned}
$$

where $\left(\vec{n}_{3}\right)^{a}=\delta^{a 3}$ is the unit vector in the isospin space, $a=1,2,3$. The frequencies of the charged fields acquire the shift $\varepsilon \rightarrow \mu-V$ for negatively charged fields ( $e, \mu^{-}$and $\rho^{-}$in our case) and $\varepsilon \rightarrow \mu+V$ for positively charged fields (for proton).

The density of the total thermodynamic potential is as follows:

$$
\begin{aligned}
\Omega= & \Omega_{N}+\Omega_{M}+\Omega_{V}+\Omega_{e}+\Omega_{\mu}, \\
\Omega_{N}= & \left(\int_{0}^{p_{\mathrm{F}, n}}+\int_{0}^{p_{\mathrm{F}, p}}\right) \frac{\mathrm{d} p p^{2}}{\pi^{2}} \sqrt{m_{N}^{2} \Phi_{N}^{2}(f)+p^{2}}-n_{n} \nu_{n}-n_{p} \nu_{p}, \\
& \nu_{p}=\mu_{n}-\mu_{e}+V-g_{\omega} \chi_{\omega} \omega_{0}-\frac{1}{2} g_{\rho} \chi_{\rho} \rho_{0}^{(3)}, \\
& \nu_{n}=\mu_{n}-g_{\omega} \chi_{\omega} \omega_{0}+\frac{1}{2} g_{\rho} \chi_{\rho} \rho_{0}^{(3)}, \\
\Omega_{M}= & \frac{1}{2}(\nabla \sigma)^{2}+\frac{1}{2} m_{\sigma}^{2} \Phi_{\sigma}^{2}+U(\sigma) \\
- & \frac{1}{2}(\nabla \omega)^{2}-\frac{1}{2} m_{\omega}^{2} \Phi_{\omega}^{2}-\frac{1}{2}\left(\nabla \rho_{0}^{(3)}\right)^{2}-\frac{1}{2} m_{\rho}^{2} \Phi_{\rho}^{2}+\Omega_{c h}^{\rho}, \\
\Omega_{c h}^{\rho}= & \left|\nabla \rho_{c}\right|^{2}+\left[m_{\rho}^{2} \Phi_{\rho}^{2}-\left(\mu_{c h}^{\rho}-V-g_{\rho} \chi_{\rho}^{\prime} \rho_{0}^{(3)}\right)^{2}\right]\left|\rho_{c}\right|^{2}, \\
\Omega_{V}= & -\frac{1}{8 \pi e^{2}}(\nabla V)^{2},
\end{aligned}
$$




$$
\Omega_{l}=\int_{0}^{p_{\mathrm{F}, l}} \frac{\mathrm{d} p p^{2}}{\pi^{2}} \sqrt{m_{l}^{2}+p^{2}}-n_{l}\left(\mu_{l}-V\right) \quad l=e, \mu,
$$

for $\left|\mu_{e}-V\right|>m_{\mu}$.

With the help of the variation

$$
\frac{\delta}{\delta n_{i}}\left(\int \Omega d \vec{r}\right)=0 \quad i=n, p, e, \mu
$$

and

$$
\frac{\delta}{\delta \xi_{i}}\left(\int \Omega d \vec{r}\right)=0, \quad \xi_{i}=\sigma, \omega, \rho_{0}^{(3)}, \rho_{c}, V
$$

we recover necessary equations of motion. In the body of the paper we used the same equations but for fields independent of coordinates.

Chemical potentials of neutrons, protons, electrons and muons are found from equations of motion (A.8):

$$
\begin{aligned}
& \mu_{n}=\sqrt{m_{N}^{2} \Phi_{N}^{2}(f)+p_{\mathrm{F}, \mathrm{n}}^{2}}+g_{\omega} \chi_{\omega} \omega_{0}-\frac{1}{2} g_{\rho} \chi_{\rho} \rho_{0}^{(3)}, \\
& \mu_{p}+V=\sqrt{m_{N}^{2} \Phi_{N}^{2}(f)+p_{\mathrm{F}, \mathrm{p}}^{2}}+g_{\omega} \chi_{\omega} \omega_{0}+\frac{1}{2} g_{\rho} \chi_{\rho} \rho_{0}^{(3)}, \\
& \mu_{l}-V=\sqrt{m_{l}^{2}+p_{\mathrm{F}, \mathrm{l}}^{2}} .
\end{aligned}
$$

Chemical potentials of proton, neutron, electron, muon and charged $\rho$ are related to each other following equilibrium conditions in reactions $n \leftrightarrow p+e$, $n \leftrightarrow p+\mu^{-}, n \leftrightarrow p+\rho^{-}$. Thus $\mu_{n}=\mu_{p}+\mu_{e}, \mu_{e}=\mu_{\mu}=\mu_{c h}^{\rho}$.

The local charge neutrality condition that we used in the paper body is now replaced by the Poisson equation for the electric potential

$$
\Delta V=-4 \pi\left(n_{p}-n_{e}-n_{\mu}+n_{c h}^{\rho}\right)
$$

where

$$
n_{e, \mu}=\frac{\left[\left(V-\mu_{e, \mu}\right)^{2}-m_{e, \mu}^{2}\right]^{3 / 2}}{3 \pi^{2}} \theta\left(\left|V-\mu_{e, \mu}\right|-m_{e, \mu}\right)
$$


for electrons and muons and

$$
n_{c h}^{\rho}=\left(g_{\rho} \chi_{\rho}^{\prime} \rho_{0}^{(3)}+V-\mu_{c h}^{\rho}\right)\left|\rho_{c}\right|^{2}<0
$$

for the charged $\rho^{-}$meson condensate. The local charge neutrality condition appears as the solution of the Poisson equation for the charged structure of the size much larger than the maximum value among Debye screening lengths of different species. For the case of Maxwell construction, solving (A.13) one may describe the boundary layer between phases. With these findings one may also solve the problem of possible occurrence of the mixed phase structures. This discussion is in full analogy to the framework of ref. [44] describing mixed phase at the first order phase transition to the s-wave negative kaon condensate.

\section{References}

[1] N.K. Glendenning, Compact Stars: Nuclear Physics, Particle Physics, and General Relativity, 2nd edition, Springer (N.Y.) (2000); F. Weber, Pulsars as Astrophysical Laboratories for Nuclear and Particle Physics, IoP Publishing, 1999.

[2] A. Akmal, V.R. Pandharipande and D.G. Ravenhall, Phys. Rev. C58 (1998) 1804 .

[3] J. Boguta and A.R. Bodmer, Nucl. Phys. A 292 (1977) 413.

[4] J. Boguta, Phys. Lett. B 106 (1981) 255.

[5] A.B. Migdal, E.E. Saperstein, M.A. Troitsky, D.N. Voskresensky, Phys. Rep. 192 (1990) 179.

[6] J.M. Lattimer, C.J. Pethick, M. Prakash, P. Haensel, Phys. Rev. Lett. 66 (1991) 2701.

[7] D.G. Yakovlev, O.Y. Gnedin, A.D. Kaminker, K.P. Levenfish, A.Y. Potekhin, Adv. Space Res. 33 (2004) 523

[8] S. Tsuruta, M.A. Teter, T. Takatsuka, T. Tatsumi, R. Tamagaki, Ap. J., 571 (2002) L143.

[9] S. Tsuruta, 2004, in: Proceedings of IAU Symposium "Young Neutron Stars and their Environments", F. Camilo, B.M. Gaensler (Eds.), vol. 218, arXiv:astro-ph/0401245.

[10] D. Blaschke, H. Grigorian, and D.N. Voskresensky, Astron. Astrophys. 424, 979 arXiv:astro-ph/0403170.

[11] E.E. Kolomeitsev and D.N. Voskresensky, Phys. Rev., C68 (2003) 015803. 
[12] I.H. Stairs, Science, 304 (2004) 547.

[13] S.E. Thorsett, and D. Chakrabarty, Ap. J. 512 (1999) 288.

[14] H. Quaintrell et al.,Astron. Astrophys., 401 (2003) 303.

[15] W. Zhang, T.E. Strohmayer and J. H. Swank, Ap. J. 482 (1997) L167; M.C. Miller, F.K. Lamb and D. Psaltis, Ap. J. 508 (1998) 791.

[16] H. Heiselberg and M. Hjorth-Jensen, eprint: astro-ph/9904214 Phys. Rep. 328 (2000) 237.

[17] D. Kaplan and A. Nelson, Phys. Lett., B175 (1986) 57.

[18] V. Koch, Int. J. Mod. Phys. E 6 (1997) 203.

[19] G.E. Brown and M. Rho, Phys. Rev. Lett. 66 (1991) 2720; Phys. Rep. 396 (2004) 1.

[20] C. Song, G.E. Brown, D.-P. Min and M. Rho, Phys. Rev., C 562244.

[21] A.A. Shanenko, E.P. Yukalova and V.I. Yukalov, Nuovo Cim. A 106 (1993) 1269; C. Song, D.P. Min and M. Rho, Phys. Lett. B 424 (1998) 226; T.S. Biro, A.A. Shanenko and V.D. Toneev, Phys. Atom. Nucl. 66 (2003) 982.

[22] I.N. Mishustin, J. Bondorf, and M. Rho, Nucl. Phys. A 555 (1993) 215;

R. Mańka and I. Bednarek, J. Phys. G: Nucl. Part. Phys. 27 (2001) 1975.

[23] K. Tuchitani et al., arXiv:nucl-th/0407004.

[24] P. Danielewicz, R. Lacey and W.G. Lynch, Science 298 (2002) 1592.

[25] T. Gaitanos, M. Di Toro, G. Ferini, M. Colonna and H.H. Wolter, arXiv:nucl-th/0402041

[26] D.N. Voskresensky, Phys. Lett. B 392 (1997) 262.

[27] M. Bando, T. Kugo, S. Vehara, K. Yamawaki and T. Yamagida, Phys. Rev. Lett. 54 (1983) 1215; M. Bando, T. Kugo, K. Yamawaki, Phys. Rep. 164 (1988) 217.

[28] A.B. Migdal, JETP Lett. 28 (1978) 35.

[29] A.D. Linde, JETP Lett. 27 (1978) 441.

[30] A.R. Bodmer, Nucl. Phys. A 526 (1991) 703.

[31] R.J. Furnstahl, H.B. Tang and B.D. Serot, Phys. Rev. C 52 (1995) 1368; R.J. Furnstahl, B.D. Serot and H.B. Tang, Nucl. Phys. A 598 (1996) 539; Nucl. Phys. A 615 (1997) 441 [Erratum-ibid. A 640 (1998) 505].

[32] B.K. Agrawal, S. Shlomo and V. Kim Au, Phys. Rev. C68 (2003) 031304.

[33] P.E. Haustein, At. Data Nucl. Data Tables 39 (1988) 185.

[34] J.D. Walecka, Ann. Phys. (N.Y.) 83 (1974) 491. 
[35] C.H. Lee, T.T.S. Kuo, G.Q. Li and G.E. Brown, Phys. Rev. C 57 (1998) 3488.

[36] J. Zimanyi and S.A. Moszkowski, Phys. Rev. C 42 (1990) 1416.

[37] R. Rapp, R. Machleidt, J.W. Durso, G.E. Brown, Phys. Rev. Lett. 82 (1999) 1827.

[38] G.Q. Li, G.E. Brown, C.H. Lee and C.M. Ko, arXiv:nucl-th/9702023

[39] M. Rho, Prog. Theor. Phys. Suppl. 149 (2003) 142 arXiv:hep-ph/0301008.

[40] M. Rho, arXiv:nucl-th/0212076

[41] N.K. Glendenning, Phys. Rev. D46 (1992) 1274.

[42] N.K. Glendenning and J. Schaffner-Bielich, Phys. Rev. C60 (1999) 025803; M. Christiansen and N.K. Glendenning, eprint: astro-ph/0008207.

[43] D.N. Voskresensky, M. Yasuhira, T. Tatsumi, Phys. Lett. B541 (2002) 93; Nucl. Phys. A723 (2003) 291.

[44] T. Maruyama, T. Tatsumi, D.N. Voskresensky, T. Tanigawa, S. Chiba, arXivenucl-th/0311076

[45] D. Blaschke, H. Grigorian, D.N. Voskresensky, arXiv:astro-ph/0403171.

[46] G.E. Brown, K. Kubodera, D. Page, P. Pizzochero, Phys. Rev. D37 (1988) 2042.

[47] D.N. Voskresensky, A.V. Senatorov, JETP Lett. 40 (1984) 1212; Sov. Phys. JETP 63 (1986) 885.

[48] D.N. Voskresensky, in book: "Physics of Neutron Star Interiors", Lecture Notes in Physics, Eds. D. Blaschke, N.K. Glendenning, A. Sedrakian, Springer, Heidelberg (2001), p. 467-502.

[49] A.B. Migdal."Theory of finite Fermi systems and properties of nuclei" (in Russian), Nauka, Moscow, 1983.

[50] E.E. Saperstein, S.V. Tolokonnikov, JETP Lett., 68 (1998) 553.

[51] A. Schwenk, B. Friman, Phys. Rev. Lett., 92 (2004) 082501.

[52] C. Schaab, D. Voskresensky, A.D. Sedrakian, F. Weber, M.K. Weigel, Astron. Astrophys. 321 (1997) 591.

[53] D.N. Voskresensky, Nucl. Phys. A555 (1993) 293.

[54] E.E. Kolomeitsev, B. Kämpfer and D. N. Voskresensky, Int. J. Mod. Phys. E 5 (1996) 313.

[55] Z. Fodor, and S.D. Katz, JHEP 0203 (2002) 014; F. Csikor, G.I. Egri, Z. Fodor, S.D. Katz, K.K. Szabo, and A.I. Tothet, hep-lat/0301027; F. Karsch, and E. Laermann, hep-lat/0305025. 